\title{
Higgs picture of the QCD-vacuum
}

\author{
C. Wetterich ${ }^{1}$ \\ Institut für Theoretische Physik \\ Universität Heidelberg \\ Philosophenweg 16, D-69120 Heidelberg
}

November 19, 2018

\begin{abstract}
The functional integral for QCD is reformulated by introducing explicitly an integration over the fluctuations of composite quarkantiquark bound states. Chiral symmetry breaking by the color singlet scalar field induces masses for the fermions. Our formulation with scalar fluctuations may be useful for lattice gauge theories by modifying the spectrum of the Dirac operator in the vacuum and permitting a simple connection to chiral perturbation theory. We propose that a "condensate" of quark-antiquark bound states in the color octet channel generates masses of the gluons by the Higgs mechanism. A simple effective action for quarks, gluons and (composite) scalars yields a surprisingly good description of the charges, masses and interactions of all low mass physical excitations - baryons, pseudoscalars and vector mesons. Dressed quarks appear as baryons and dressed gluons as vector mesons.
\end{abstract}

\footnotetext{
${ }^{1}$ e-mail: C.Wetterich@thphys.uni-heidelberg.de
} 


\section{Introduction}

An analytic description of the vacuum in QCD remains a central goal in quantum field theory. We have witnessed convincing progress of numerical simulations of QCD on a lattice. Still, even an approximate analytic understanding would be a highly valuable complement. Computations of strong cross sections and decay rates are very hard in lattice QCD. Simulations have a long way to go before the properties of nuclei can be explained. Furthermore, a huge present and future experimental program tries to gather information about the QCD phase transition and the phase diagram as function of baryon density and temperature. Simulations for high baryon density are notoriously difficult and an analytical understanding would be very helpful.

A key issue for the simplicity and success of an analytical description is an efficient description of the relevant degrees of freedom. In QCD the relevant degrees of freedom depend on the momentum scale. Processes involving high momenta (more precisely high virtuality) are well described by quarks and gluons. Perturbation theory based on the microscopic action for quarks and gluons with a small gauge coupling yields reliable results. In contrast, the relevant degrees of freedom at long distances or small momenta are mesons and baryons. An efficient description of the vacuum should therefore involve degrees of freedom for the low mass mesons. Typically, the lowest mass excitations in the QCD vacuum should comprise the pseudoscalar meson octet and singlet $\left(\eta^{\prime}\right)$, the vector meson octet and the baryon octet in the respective channels. We observe that this spectrum of real QCD differs strongly from pure QCD (gluodynamics) without quarks where the low excitations consist of glueballs. This simple fact suggests that an efficient analytic description of real QCD at low momentum differs substantially from gluodynamics. As the momentum scale is lowered an analytic description of real QCD should effectively switch from gluons and quarks to mesons and baryons.

The most prominent long distance degrees of freedom are scalar quarkantiquark $(\bar{q} q)$ bound states. The expectation value of such a composite scalar field induces spontaneous chiral symmetry breaking. The excitations of this scalar field describe then the associated (pseudo-) Goldstone bosons $\pi, K, \eta$ as well as the $\eta^{\prime}$. Our first task for an analytic description will therefore be to supplement quarks and gluons by the degrees of freedom for composite scalar (and pseudoscalar) meson fields. In the first part of this work (sect. 2) we will reformulate the functional integral for QCD in an exactly equivalent form which comprises an integration over explicit scalar degrees of freedom. These scalars are of minor importance at high momenta but become crucial for the properties of the vacuum. Besides the analytical advantage of a simple connection to chiral perturbation theory this reformulation may also 
offer important benefits for lattice simulations. The spectrum of the Dirac operator acquires a mass gap even in the chiral limit and the contact to chiral perturbation theory at long distances should become straightforward.

Concerning the QCD phase transition the task for an analytical description becomes even more involved: the formalism should now describe simultaneously quarks, gluons, mesons and baryons. Above the critical temperature a quark gluon plasma is a valid approximation and explicit mesons or baryons play no important role. In the hadron gas below the critical temperature the mesons (and baryons) dominate whereas quarks and gluons become irrelevant. A simple analytical description has to capture all these degrees of freedom and provide for a mechanism explaining why their relative importance changes abruptly as a function of temperature.

Associating spontaneous chiral symmetry breaking with the phase transition yields a simple explanation why the quarks disappear from the relevant spectrum below the critical temperature. $T_{c}$ : the fermions get massive for $T<T_{c}$. What is needed is a similar mechanism for the gluons. For the electroweak phase transition the effective generation of a mass for the $W$ and $Z$-bosons is well known. As the universe cools down below the critical temperature both the gauge bosons and the fermions acquire a mass due to the Higgs mechanism associated to the "spontaneous breaking" of the electroweak gauge symmetry. The second part of this work will review a similar "Higgs mechanism" for QCD, namely the "spontaneous breaking of color" by the expectation value of composite $\bar{q} q$-scalars in the color-octet channel [1].

Strictly speaking, local symmetries cannot be broken spontaneously in the vacuum. This has led to the realisation that the confinement and the Higgs description are not necessarily associated to mutually exclusive phases. They may only be different facets [3] of one and same physical state ${ }^{2}$ We stress that this observation is not only of formal importance. For example, the high temperature phase transition of electroweak interactions with a small Higgs scalar mass ends for a larger scalar mass in a critical endpoint. Beyond this endpoint the phase transition is replaced by an analytical crossover 4 . In this region - which is relevant for a realistic Higgs mass in the standard model - a Higgs and a confinement description can be used simultaneously. Our picture of the QCD vacuum resembles in many aspects the "strongly coupled electroweak theory" at high temperature ${ }^{3}$. We will present a Higgs

\footnotetext{
${ }^{2}$ The complementarity between the Higgs and confinement description has been considered earlier for toy models with fundamental colored scalar fields [2].

${ }^{3}$ Without a direct connection to the standard model the $S U(2)$-Yang-Mills theory with strong gauge coupling and fundamental scalar has been first simulated on the lattice in [5].
} 
description of QCD as a complementary picture to the usual confinement picture. Both the Higgs description and the confinement picture are considered as valid descriptions of one and the same physical properties of QCD. A valid Higgs picture has to be consistent with the well established results of the confinement picture and of lattice QCD.

With respect to color the composite scalar $\bar{q} q$-bilinears transform as singlets and octets, each in the $(\overline{3}, 3)$-representation of the $S U(3)_{L} \times S U(3)_{R}$ chiral flavor symmetry. We will explore the hypothesis that the scalar octets induce "spontaneous color symmetry breaking" while a "physical" global $S U(3)$ symmetry is preserved. This global symmetry can be used to classify the spectrum of excitations according to the "eightfold way". We find that all gluons acquire a mass by the "Higgs mechanism" and belong to an octet of the physical $S U(3)$-symmetry. Our picture provides an effective infrared cutoff for real QCD by mass generation. (The cutoff for gluodynamics is expected to be different!)

The Higgs mechanism also gives integer electric charge and strangeness to all physical particles according to their $S U(3)$-transformation properties. Furthermore, the expectation value of the quark-antiquark color octet breaks the global chiral symmetry. In consequence the fermions become massive and the spectrum contains light pions and kaons as pseudo-Goldstone bosons. In the limit of equal masses for the three light quarks the global vector-like SU(3)-symmetry of the "eightfold way" becomes exact. The nine quarks transform as an octet and a singlet. We identify the octet with the low mass baryons - this is quark-baryon duality. The singlet is associated with the $\Lambda$ (1405) baryon and has parity opposite to the nucleons. Similarly, the eight gluons carry the quantum numbers of the light vector mesons $\rho, K^{*}, \omega$. The identification of massive gluons with the vector mesons is called gluon-meson duality.

We propose that the main nonperturbative new ingredient for the effective action of low-momentum QCD consists of scalar fields representing quarkantiquark bound states. Once these composite operators are treated on the same footing as the quark and gluon fields, the description of propagators and vertices becomes again very simple. We will investigate the phenomenological consequences of an effective action that adds to the usual gluon and quark (gauge covariant) kinetic terms the corresponding kinetic terms for the scalars with quantum numbers of the $\bar{q} q$-composites. Furthermore, this is supplemented by a scalar potential and a Yukawa interaction between quarks and scalars. It is very remarkable that such a simple effective action can account for the quantum numbers, the masses and the interactions of the pseudoscalar octet and the $\eta^{\prime}$ mesons, the vector meson octet and the baryon octet! Once the free parameters of this effective action are fixed by match- 
ing observation, several nontrivial properties of hadrons can be "predicted" without further unknown parameters. These findings suggest that the longsought dual description of long-distance strong interactions can be realized by the addition of fields for composites. The situation would then be quite similar to the asymptotically free nonlinear sigma model in two dimensions where the addition of the composite "radial excitation" provides for a simple dual description of the low momentum behavior [6].

Spontaneous breaking of color has also been proposed [7] for situations with a very high baryon density, as perhaps in the interior of neutron stars. In this proposal a condensation of diquark operators is responsible for color superconductivity and spontaneous breaking of baryon number. In particular, the suggestion of color-flavor locking [8] offers analogies to our description of the vacuum, even though different physical situations are described (vacuum vs. high density state) and the pattern of spontaneous color-symmetry breaking is distinct (quark-antiquark vs. quark-quark condensate; conserved vs. broken baryon number). This analogy may be an important key for the understanding of possible phase transitions to a high density phase of QCD.

In the second part of this work we first present in sect. 3 our proposal for a simple effective action for QCD. Sect. 4 describes the "Higgs picture" with a non-vanishing expectation value of the scalar octet field. The gauge invariant description in terms of nonlinear fields is introduced in sect. 5. Sect. 6 discusses the role of a hidden local symmetry in the nonlinear description which will permit a direct connection to ideas of "vector dominance". The elctromagnetic interactions can be used as an efficient probe of our picture since no new free parameters are introduced (sect. 7). In sect. 8 we turn to the interactions of the vector mesons and the decay $\rho \rightarrow 2 \pi$. Here we also make direct contact with the description of vector mesons as gauge invariant $\bar{q} \gamma^{\mu} q$ bound states in sect. 2. Sect. 9 discusses the interactions of the pseudoscalar mesons. We will see that the strength of the vector and axialvector couplings of the nucleons as well as the pion interactions beyond leading order chiral perturbation are successfully accounted for by our simple

effective action. Sect. 10 finally presents conclusions and discussion and makes a simple proposal for the QCD phase diagram.

\section{Functional integral with composite fields}

Our starting point is the partition function for QCD

$$
Z=\int \mathcal{D} \psi \mathcal{D} A e^{-S}
$$


where $S$ is the gauge invariant classical action for quarks and gluons. Here the quarks are described by Grassmann variables obeying $\{\psi(x), \psi(y)\}=$ $0,\left\{\psi(q), \psi\left(q^{\prime}\right)\right\}=0$ in position and momentum space, respectively. The difficult part in the functional integral (11) is the functional measure $\int \mathcal{D} \psi \mathcal{D} A$ which includes the regularization, for example on a lattice, or the gauge fixing and ghost parts for a continuum formulation. We do not need a specification here and only assume that the functional measure preserves the gauge symmetry.

It is well known that local gauge symmetries cannot be spontaneously broken (in a strict sense). Therefore only gauge invariant quantities can have nonzero expectation values. A prominent example concerns the correlation functions for scalars and pseudoscalars which are contained in

$$
G_{s}(x, y)=-\left\langle\left(\bar{\psi}_{L}(x) \psi_{R}(x)\right)\left(\bar{\psi}_{R}(y) \psi_{L}(y)\right)\right\rangle \text {. }
$$

Here $\left(\bar{\psi}_{R} \psi_{L}\right)=\left(\bar{\psi} \frac{1+\gamma^{5}}{2} \psi\right)$ and brackets denote contractions of spinor and color indices, while we have not displayed the (open) flavor indices. For the example of the pion channel the two point function decays for large $|x-y|$ as $G_{s} \sim \exp \left(-m_{\pi}|x-y|\right)$ and this is the way how the pion mass is measured, for example on the lattice. Similarly, the $\rho$-meson mass can be extracted from the gauge invariant correlation function in the vector channel

$$
G_{V}^{\mu \nu}(x, y)=\left\langle\left(\bar{\psi}(x) \gamma^{\mu} \psi(x)\right)\left(\bar{\psi}(y) \gamma^{\nu} \psi(y)\right)\right\rangle .
$$

A convenient way for the computation of correlation functions for gauge invariant quark-antiquark bilinears is the introduction of gauge invariant sources

$$
S_{j}=-\int d^{4} x\left\{\left(\bar{\psi}_{L}(x) j_{s}^{\dagger}(x) \psi_{R}(x)+\text { h.c. }\right)+\bar{\psi}(x) \gamma^{\mu} j_{V_{\mu}}(x) \psi(x)\right\} .
$$

Here $j_{s}$ is a complex $N_{f} \times N_{f}$ matrix and $j_{V}$ denotes a hermitean $N_{f} \times N_{f}$ matrix, with $N_{f}$ the number of quark flavors. We will concentrate here on the three light flavors of quarks $\left(N_{f}=3\right)$ while considering the two flavor case as a pedagogical example below. Then the physical values of the scalar sources are given by the current quark masses

$$
j_{s}=j_{s}^{\dagger}=m_{q}=\left(\begin{array}{ccc}
m_{u} & & \\
& m_{d} & \\
& & m_{s}
\end{array}\right)
$$

while the vector source vanishes $j_{V}=0$. Adding $S_{j}$ to $S$ the partition function $Z[j]$ (11) becomes now a functional of the sources. 
As an example, we may write the expectation value of the scalar quarkantiquark bilinear as $(a, b$ are flavor indices while color and spinor indices are contracted)

$$
\left\langle\bar{\psi}_{L b}(x) \psi_{R a}(x)\right\rangle=\sigma_{a b}(x)=Z^{-1} \frac{\delta Z}{\delta j_{a b}^{*}(x)} .
$$

Similarly, the (unconnected) two point function obtains from the matrix of the second functional variations ${ }^{4}$

$$
\begin{aligned}
G_{s}^{(u)}(x, y) & =-\left\langle\left(\bar{\psi}_{R}(x) \psi_{L}(x)\right)\left(\bar{\psi}_{L}(y) \psi_{R}(y)\right)\right\rangle \\
& =Z^{-1} \frac{\delta^{2} Z}{\delta j(x) \delta j^{*}(y)} .
\end{aligned}
$$

As usual, the expectation value or the connected Green's function can be derived from

$$
W[j]=\ln Z[j]
$$

as

$$
\sigma_{a b}(x)=\frac{\delta W}{\delta j_{a b}^{*}(x)}
$$

or

$$
G_{s}(x, y)=G_{s}^{(u)}(x, y)-\sigma^{*}(x) \sigma(y)=\frac{\delta^{2} W}{\delta j(x) \delta j^{*}(y)} .
$$

Finally, the effective action $\Gamma$ is a functional of the "classical field" $\sigma$

$$
\Gamma[\sigma]=-W[j]+\int d^{4} x \operatorname{tr}\left(j^{\dagger}(x) \sigma(x)+\sigma^{\dagger}(x) j(x)\right)
$$

where $\sigma$ corresponds to a given source $j$ and $j=j[\sigma]$ is computed by inversion of

$$
\sigma[j]=\frac{\delta W}{\delta j^{*}} .
$$

The field equation reads

$$
\frac{\delta \Gamma}{\delta \sigma_{a b}(x)}=j_{a b}^{*}(x)
$$

Furthermore, the second functional variation of $\Gamma$ with respect to $\sigma$ equals the inverse connected two point function, i.e. the inverse of the second functional variation of $W$ with respect to $j$

$$
\Gamma^{(2)} W^{(2)}=\mathbb{1} .
$$

\footnotetext{
${ }^{4}$ In flavor space $\left(G_{s}\right)_{a b, c d}$ is a $N_{f}^{2} \times N_{f}^{2}$ matrix, with rows and columns labeled by flavor index pairs $(a b)$ and $(c d)$.
} 
(Note that for this matrix notation all internal and space or momentum labels of $\sigma$ are collected into a vector.) Of course, this setting can be generalized to other gauge invariant composite operators, like vector fields, in a straightforward way.

Let us concentrate here on the scalar sector and, for simplicity of the demonstration, on $N_{f}=2$. For $x$-independent values $\sigma(x)=\sigma$ one has $\Gamma=\int d^{4} x U(\sigma)$ where the effective potential $U$ is now a simple function of the complex $2 x 2$ matrices $\sigma$. By construction $U(\sigma)$ is invariant under the chiral flavor rotations $S U\left(N_{f}\right)_{L} \times S U\left(N_{f}\right)_{R}$. If we expand $U(\sigma)$ in powers of $\sigma$ only invariants can appear

$$
\begin{aligned}
U(\sigma)= & m^{2} \operatorname{tr}\left(\sigma^{\dagger} \sigma\right)-\frac{\nu}{2}\left(\operatorname{det} \sigma+\operatorname{det} \sigma^{\dagger}\right) \\
& +\frac{\lambda_{1}}{2}\left(\operatorname{tr}\left(\sigma^{\dagger} \sigma\right)\right)^{2}+\frac{\lambda_{2}}{2} \operatorname{tr}\left(\sigma^{\dagger} \sigma-\frac{1}{2} \operatorname{tr}\left(\sigma^{\dagger} \sigma\right)\right)^{2}+\ldots
\end{aligned}
$$

and we note that the axial $U(1)_{A}$-anomaly is reflected in the term $\sim \nu$. The expectation value of $\sigma$ follows from the field equation

$$
\frac{\partial U}{\partial \sigma}=j^{*}=m_{q}
$$

This suggests to define

$$
U_{j}=U-\operatorname{tr}\left(j^{\dagger} \sigma+\sigma^{\dagger} j\right) \quad, \quad \frac{\partial U_{j}}{\partial \sigma}=0 .
$$

In particular, for equal up-and-down-quark masses the minimum of $U_{j}$ occurs typically for

$$
\langle\sigma\rangle=\left(\begin{array}{cc}
\bar{\sigma} & \\
& \bar{\sigma}
\end{array}\right)
$$

and breaks the chiral $S U(2)_{L} \times S U(2)_{R}$ symmetry to a vectorlike "diagonal" $S U(2)_{V}$-symmetry. We recall that $\bar{\sigma}$ is directly related to the quark-antiquark condensate

$$
\bar{\sigma}=-\frac{1}{2}\langle\bar{\psi} \psi\rangle .
$$

One of the advantages of our formulation is the explicit chiral symmetry of $U(\sigma)$ independently of the quark masses, since $m_{q}$ enters only through the source term in the field equation (16). In particular, one can make direct contact to chiral perturbation theory by representing the pseudoscalar (pseudo-) Goldstone bosons by a nonlinear field ${ }^{5} U(x)$

$$
\sigma(x)=\bar{\sigma} U(x), U^{\dagger} U=1 .
$$

\footnotetext{
${ }^{5}$ The coincidence of the symbol $U$ for the nonlinear chiral field and the effective potential is unfortunate but kept here for the sake of agreement with widely used conventions.
} 
Inserting the nonlinear field (20) into the effective potential (17) yields

$$
U_{j}(U)=\text { const }-m_{q} \bar{\sigma} t r\left(U+U^{\dagger}\right)-\frac{\nu}{2} \bar{\sigma}^{2}\left(\operatorname{det} U+\operatorname{det} U^{\dagger}\right) .
$$

In the chiral limit $m_{q}=0$ the potential only involves the $\eta$ meson - the chiral $U(1)_{A}$-anomaly produces a mass term for the $\eta$-meson. In this limit the pions are massless Goldstone bosons. For nonvanishing quark masses also the pions acquire a mass $m_{\pi}^{2} \sim m_{q}$.

Let us neglect the $\eta$-meson and discuss explicitly the interactions of the pions

$$
\operatorname{det} U=1, U(x)=\exp \left\{\frac{i \vec{\tau} \vec{\pi}(x)}{f_{\pi}}\right\} .
$$

The kinetic term for $\sigma$ results in the nonlinear kinetic term for the pions

$$
\bar{Z} \operatorname{tr}\left(\partial^{\mu} \sigma^{\dagger} \partial_{\mu} \sigma\right) \rightarrow \bar{Z} \bar{\sigma}^{2} \operatorname{tr}\left(\partial^{\mu} U^{\dagger} \partial_{\mu} U\right)=\frac{2 \bar{Z} \bar{\sigma}^{2}}{f_{\pi}^{2}} \partial^{\mu} \vec{\pi} \partial_{\mu} \vec{\pi}+\ldots
$$

where we identify the pion decay constant

$$
f_{\pi}=2 \bar{Z}^{1 / 2} \bar{\sigma}
$$

The effective action takes now the form familiar from chiral perturbation theory

$$
\mathcal{L}[U]=\frac{f_{\pi}^{2}}{4}\left\{\operatorname{tr}\left(\partial^{\mu} U^{\dagger} \partial_{\mu} U\right)-2 B m_{q} \operatorname{tr}\left(U+U^{\dagger}\right)+\ldots\right\}
$$

with

$$
B=\frac{2 \bar{\sigma}}{f_{\pi}^{2}}=-\frac{\langle\bar{\psi} \psi\rangle}{f_{\pi}^{2}} .
$$

In this treatment, however, $\mathcal{L}[U]$ is already the effective Lagrangian from which the 1PI-vertices follow directly by taking suitable derivatives with respect to the fields. No more fluctuations have to be incorporated at this stage. In particular, there are no explicit meson fluctuations.

In order to recover chiral perturbation theory we want to reformulate our problem such that explicit pion fluctuations are incorporated in the computation of the effective action. Indeed, it is possible to reformulate the original functional integral for QCD (II) into an exactly equivalent functional integral which now involves explicitly an integration also over scalar fluctuations. This is achieved by means of a Hubbard-Stratonovich transformation [9]. Let us denote the scalar fermion bilinears by

$$
\tilde{\sigma}_{a b}=\bar{\psi}_{L b} \psi_{R a},\left\langle\tilde{\sigma}_{a b}\right\rangle=\sigma_{a b}
$$


We next introduce a unit into the functional integral (1)

$$
\mathbb{1}=N \int \mathcal{D} \sigma^{\prime} \exp \left\{-\int \frac{d^{4} q}{(2 \pi)^{4}} \operatorname{tr}\left\{\left(\sigma^{\prime}-\lambda_{\sigma} \tilde{\sigma}-j\right)^{\dagger} \lambda_{\sigma}^{-1}\left(\sigma^{\prime}-\lambda_{\sigma} \tilde{\sigma}-j\right)\right\}\right\}
$$

where $N$ is an irrelevant normalization constant. The functional integral for the partition function $Z[j]$ involves now an additional integration over the scalar field $\sigma^{\prime}$

$$
Z[j]=\int \mathcal{D} \psi \mathcal{D} A \mathcal{D} \sigma^{\prime} \exp \left(-S_{j}^{(\sigma)}\right) .
$$

Here the action $S_{j}$ in eq. (4) is replaced by $S_{j}^{(\sigma)}$, i.e.

$$
\begin{aligned}
S_{j}^{(\sigma)}= & S_{0}+\int \frac{d^{4} q}{(2 \pi)^{4}} \operatorname{tr}\left\{{\sigma^{\prime}}^{\dagger} \lambda_{\sigma}^{-1} \sigma^{\prime}-\left(\sigma^{\prime \dagger} \tilde{\sigma}+\tilde{\sigma}^{\dagger} \sigma^{\prime}\right)+\lambda_{\sigma} \tilde{\sigma}^{\dagger} \tilde{\sigma}\right. \\
& \left.-\left(j^{\dagger} \lambda_{\sigma}^{-1} \sigma^{\prime}+\sigma^{\prime \dagger} \lambda_{\sigma}^{-1} j\right)+j^{\dagger} \lambda_{\sigma}^{-1} j\right\}
\end{aligned}
$$

where $S_{0}$ is the QCD-action for quarks and gluons without the quark mass term. Indeed, the quark mass term $\sim \operatorname{tr}\left(j^{\dagger} \widetilde{\sigma}\right)$ is canceled by a corresponding term from eq. (28) such that the nonvanishing quark masses appear now as a source term multiplying the scalar field $\sigma^{\prime}$.

In eq. (28) $\sigma^{\prime}$ stands for $\sigma_{a b}^{\prime}(q)$ and $\lambda_{\sigma}^{-1}(q)$ is an arbitrary positive function of $q^{2}$ such that the Gaussian integral is well defined and rotation symmetry preserved. If we choose

$$
\lambda_{\sigma}=\frac{1}{M^{2}+Z q^{2}}
$$

we can identify the term $\sigma^{\prime \dagger} \lambda_{\sigma}^{-1} \sigma^{\prime}$ with a kinetic and mass term for $\sigma^{\prime}$ such that $\lambda_{\sigma}$ corresponds to the "classical scalar propagator". The term $\tilde{\sigma}^{\dagger} \sigma^{\prime}$ denotes a Yukawa coupling of the $\sigma^{\prime}$ field to the quarks. Finally, the expression $\lambda_{\sigma} \tilde{\sigma}^{\dagger} \tilde{\sigma}$ denotes a four quark interaction that is nonlocal for $Z \neq 0$.

The explicit four-quark-interaction is cumbersome and we may wish to omit it. This can be done if we introduce into the original action for quarks and gluons an additional four-quark-interaction with the opposite sign - this is then canceled by the piece arising from the Hubbard-Stratonovich transformation. At first sight, this seems to be a high prize to pay since we are not dealing any more with the standard QCD action where the quarks interact only via gluon exchange. With a second look, however, this is no problem. Implementing this modified QCD action for lattice simulations will even result in an "improved" QCD action if suitable values for $M^{2}$ and $Z$ are chosen in eq. (31).

The basic ingredient for this argument is universality. In QCD the precise form of the short distance action (classical action) is actually irrelevant, 
provided this action belongs to the universality class of QCD. Independently of the precise form of the classical action the long distance behavior will then only depend on the value of the renormalized gauge coupling (which sets the scale via dimensional transmutation) and the quark masses. This fact is widely used in the form of "improved actions" for lattice QCD. In a qualitative sense the mapping of the continuous QCD onto a lattice with finite lattice spacing $a$ involves an integration of fluctuations with momenta $q^{2}>\pi^{2} / a^{2}$. Performing the gluon integration in perturbation theory the box diagrams with exchange of two gluons indeed lead to four-quark-interactions in the form needed here (plus others) [10, 11. Their strength can be used to optimize $M^{2}$ and $Z$ for a given lattice spacing $a$, thus improving the action. We emphasize, however, that due to universality this improvement is not necessary, in principle. The precise values of $M^{2}$ and $Z$ should not be important as long as $M^{2}$ is not too small and $Z$ not too large such that the interaction remains "essentially local" and within the universality class of QCD.

In the formulation with additional scalars the issue of the correct universality class actually needs some consideration. For very small $M^{2}$ or very large quartic coupling $\lambda_{\sigma}$ (in units of $a$ ) the theory will be in a different universality class where the scale of chiral symmetry breaking is set by $\lambda_{\sigma}$ and not by $\Lambda_{Q C D}$ as appropriate for QCD. This issue is discussed quantitatively in [11. In particular, for too small $M^{2}$ the model contains an additional relevant parameter beyond the gauge coupling (and quark masses). ${ }^{6}$

We suggest that lattice simulations with explicit meson degrees of freedom and the action (30) (without the quartic term $\sim \lambda_{\sigma} \tilde{\sigma}^{\dagger} \tilde{\sigma}$ and for appropriate $M^{2}, Z$ ) may actually permit a direct access to the chiral limit of vanishing quark masses. In the current formulation (without explicit meson degrees of freedom) one of the main obstacles for the simulation of the chiral limit is the complicated spectrum of the Dirac operator for the quarks. In presence of meson fields the dominant configurations would correspond to non-zero values of $\sigma^{\prime}$. In term, this induces a mass gap for the Dirac operator due to the Yukawa coupling $\tilde{\sigma}^{\dagger} \sigma^{\prime}$. This should make simulations with dynamical quarks much simpler. In addition, the meson fluctuations provide the degrees of freedom appearing in chiral perturbation in a very explicit way. The contact to chiral perturbation theory should therefore be facilitated.

Our formulation where the source term appears explicitly in the transformation (28) has the additional advantage that the quark masses only appear

${ }^{6}$ This also tells us that QCD lattice simulations with explicit scalar fields are only meaningful with dynamical quarks. In the quenched approximation the scalar sector decouples from the gluons and therefore induces the relevant parameters of a pure scalar theory that should not be present for the QCD universality class. 
as terms linear in the scalar field. On the level of the effective action we can perform a single computation for all values of the quark masses and fully exploit the chiral symmetry. The quark masses appear then only as source terms in the field equations. Still, we have to take into account the modifications due to the term $j^{\dagger} \lambda_{\sigma}^{-1} j$ in eq. (30). This modifies the relation between the expectation value of $\sigma^{\prime}$ and $\tilde{\sigma}$ according to

$$
\frac{\delta W}{\delta j^{*}}=\left\langle\lambda_{\sigma}^{-1}\left(\sigma^{\prime}-j\right)\right\rangle=\langle\tilde{\sigma}\rangle=\sigma
$$

such that

$$
\varphi=\left\langle\sigma^{\prime}\right\rangle=\lambda_{\sigma} \sigma+j
$$

We may bring the functional integral even closer to the standard formulation for a theory with quarks, gluons and scalars by using standard sources of $\sigma^{\prime}$

$$
j_{\varphi}=\lambda_{\sigma}^{-1} j
$$

and subtracting the term quadratic in $j$ which does not involve the fields

$$
S_{j}^{(\varphi)}=S_{j}^{(\sigma)}-\int j^{\dagger} \lambda_{\sigma}^{-1} j
$$

In this formulation, the generating functional for the connected Greens functions is related to eq. (8) by

$$
W_{\varphi}=W+\int j^{\dagger} \lambda_{\sigma}^{-1} j
$$

and the effective action is defined as usual

$$
\Gamma[\varphi]=-W_{\varphi}+\int\left(j_{\varphi}^{\dagger} \varphi+\varphi^{\dagger} j_{\varphi}\right)
$$

Comparing with $\Gamma[\sigma]$ (11) one obtains

$$
\Gamma[\varphi]=\Gamma[\sigma]+\int \frac{\delta \Gamma}{\delta \sigma} \lambda_{\sigma}^{-1} \frac{\delta \Gamma}{\delta \sigma} .
$$

(We have not used different symbols, but it should be clear that $\Gamma[\varphi]$ and $\Gamma[\sigma]$ are not related simply by a variable transformation.) The difference between $\Gamma[\varphi]$ and $\Gamma[\sigma]$ vanishes at the extrema of $\Gamma[\sigma]$ but modifies, for example, the behavior for large values of the fields. Typically, $\Gamma[\varphi]$ is a better behaved quantity. For example, the effective potential is bounded from below which is not guaranteed for $\Gamma[\sigma]$. The field equations derived from $\Gamma[\varphi]$ take the standard form

$$
\frac{\delta \Gamma}{\delta \varphi}=j_{\varphi}^{\dagger}=\lambda_{\sigma}^{-1} j^{\dagger}
$$




\section{QCD effective action with composite scalar fields}

Let us next discuss the effective action for QCD in a formulation where explicit scalar fields for composite $\bar{q} q$ bilinears are used according to the preceding section. We will see that a very simple form of the effective action can account for a great deal of the strong interaction phenomenology in a surprisingly simple fashion. By definition the effective action generates the one-particle-irreducible (1PI) correlation functions and includes all quantum fluctuations. It therefore contains the direct information about the propagators and proper vertices.

In addition to the quark and gluon fields we consider scalar fields with the transformation properties of quark-antiquark pairs. With respect to the color and chiral flavor rotations $S U(3)_{C} \times S U(3)_{L} \times S U(3)_{R}$ the three light left-handed and right-handed quarks $\psi_{L}, \psi_{R}$ transform as $(3,3,1)$ and $(3,1,3)$, respectively. Quark-antiquark bilinears therefore contain a color singlet $\Phi(1, \overline{3}, 3)$ and a color octet $\chi(8, \overline{3}, 3)$

$$
\begin{aligned}
\gamma_{i j} & =\chi_{i j}+\frac{1}{\sqrt{3}} \phi \delta_{i j}, \\
\phi & =\frac{1}{\sqrt{3}} \gamma_{i i}, \quad \chi_{i i}=0 .
\end{aligned}
$$

Here we use a matrix notation for the flavor indices and write the color indices $i, j$ explicitly, e.g. $\chi_{i j, a b} \equiv \chi_{i j}, \phi_{a b} \equiv \phi$. Then $\gamma_{i j}$ contains 81 complex scalar fields. Similarly, the quark fields are represented as three flavor vectors $\psi_{a i} \equiv \psi_{i}, \bar{\psi}_{i a} \equiv \bar{\psi}_{i}$. As usual we represent the eight $S U(3)_{C}$-gauge fields by

$$
A_{i j, \mu}=\frac{1}{2} A_{\mu}^{z}\left(\lambda_{z}\right)_{i j}
$$

where $\lambda_{z}$ are the eight Gell-Mann matrices normalized according to $\operatorname{Tr}\left(\lambda_{y} \lambda_{z}\right)=$ $2 \delta_{y z}$.

We consider a very simple effective Lagrangian containing only terms with dimension up to four ${ }^{7}$

$$
\begin{aligned}
\mathcal{L}= & i Z_{\psi} \bar{\psi}_{i} \gamma^{\mu} \partial_{\mu} \psi_{i}+g Z_{\psi} \bar{\psi}_{i} \gamma^{\mu} A_{i j, \mu} \psi_{j}+\frac{1}{2} G_{i j}^{\mu \nu} G_{j i, \mu \nu} \\
& +\operatorname{Tr}\left\{\left(D^{\mu} \gamma_{i j}\right)^{\dagger} D_{\mu} \gamma_{i j}\right\}+U(\gamma) \\
& +Z_{\psi} \bar{\psi}_{i}\left[\left(h \phi \delta_{i j}+\tilde{h} \chi_{i j}\right) \frac{1+\gamma_{5}}{2}-\left(h \phi^{\dagger} \delta_{i j}+\tilde{h} \chi_{j i}^{\dagger}\right) \frac{1-\gamma_{5}}{2}\right] \psi_{j} .
\end{aligned}
$$

\footnotetext{
${ }^{7}$ For our Euclidean conventions for fermions see 12 .
} 
Here $G_{i j, \mu \nu}=\partial_{\mu} A_{i j, \nu}-\partial_{\nu} A_{i j, \mu}-i g A_{i k, \mu} A_{k j, \nu}+i g A_{i k, \nu} A_{k j, \mu}$ and the interaction between gluons and $\chi$ arise from the covariant derivative

$$
D_{\mu} \gamma_{i j}=\partial_{\mu} \gamma_{i j}-i g A_{i k, \mu} \gamma_{k j}+i g \gamma_{i k} A_{k j, \mu}
$$

In our notation the transposition acts only on flavor indices, e.g. $\left(\gamma_{i j}^{\dagger}\right)_{a b}=$ $\gamma_{i j, b a}^{*}$. The effective potential

$$
\begin{aligned}
& U(\gamma)=U_{0}(\chi, \phi)-\frac{1}{2} \nu\left(\operatorname{det} \phi+\operatorname{det} \phi^{\dagger}\right)-\frac{1}{2} \nu^{\prime}\left(E(\phi, \chi)+E^{*}(\phi, \chi)\right) \\
& E(\phi, \chi)=\frac{1}{6} \epsilon_{a_{1} a_{2} a_{3}} \epsilon_{b_{1} b_{2} b_{3}} \phi_{a_{1} b_{1}} \chi_{i j, a_{2} b_{2}} \chi_{j i, a_{3} b_{3}}
\end{aligned}
$$

conserves the symmetry $S U(3)_{c} \times S U(3)_{L} \times S U(3)_{R}$. It is decomposed into a part $U_{0}$ which conserves also the axial $U(1)_{A}$ symmetry and an anomaly contribution. The latter is parameterized here by the 't Hooft terms [13] $\sim \nu, \nu^{\prime}$. The details of $U$ will not be relevant for our discussion except for the location of the minimum that will be characterized by two parameters $\sigma_{0}$ and $\chi_{0}$. Finally, explicit chiral symmetry breaking is induced by a linear term 14

$$
\begin{aligned}
\mathcal{L}_{j} & =-\frac{1}{2} Z_{\phi}^{-1 / 2} \operatorname{Tr}\left(j^{\dagger} \phi+\phi^{\dagger} j\right) \\
j=j^{\dagger} & =a_{q} \bar{m}=a_{q} \operatorname{diag}\left(\bar{m}_{u}, \bar{m}_{d}, \bar{m}_{s}\right)
\end{aligned}
$$

with $\bar{m}_{q}$ the current quark masses normalized at some appropriate scale, say $\mu=2 \mathrm{GeV}$. We note that the quark wave function renormalization $Z_{\psi}$ can be absorbed by a rescaling of $\psi$. We keep it here and normalize $\psi$ at a perturbative scale $\mu=2 \mathrm{GeV}$.

In general, the wave function renormalizations $Z_{\phi}, Z_{\psi}$ and the dimensionless couplings $g, h$ depend on momentum or an appropriate renormalization scale $\mu$. The phenomenological analysis will mainly concentrate on the low energy limit where we take all couplings as constants. Besides the explicit chiral symmetry breaking in (45) the phenomenology will be described in terms of the seven real parameters $g, h, \tilde{h}, \sigma_{0}, \chi_{0}, \nu$ and $\nu^{\prime}$. Here $\nu$ and $\nu^{\prime}$ will only enter the mass of $\eta^{\prime}$ and $h, \tilde{h}$ will be fixed by the baryon masses. Then three parameters $g, \sigma_{0}, \chi_{0}$ remain for the description of the pseudoscalars and vector mesons and their interactions, including the interactions with baryons. In fact, the effective action (42) is the most general ${ }^{8}$ one which contains only "renormalizable" interactions and is consistent with

${ }^{8}$ The only exception is the omission of a possible $U(1)_{A}$-violating term cubic in $\chi$ which can be found in 15 . 
$S U(3)_{C} \times S U(3)_{L} \times S U(3)_{R}$ symmetry, as well as with the discrete transformations parity and charge conjugation.

We will show that the masses and interactions of the octet of baryons as well as the pseudoscalar and vector mesons are indeed well described by the simple effective action (42), (45). We find this rather remarkable in view of the fact that we have only added rather straightforward terms for composite scalars ("scalar Vervollständigung" [1). Of course, the crucial ingredient is the nontrivial minimum of $U(\chi, \phi)$.

Actually, there is no reason why only interactions involving scalar and pseudoscalar quark-antiquark bilinears should be present as in (42). Effective four-quark interactions (1PI) in the vector or axial-vector channel are certainly induced by fluctuations, and we will discuss them below. There is also no need that the effective action has to be of the "renormalizable form" (42). In fact, we have at present no strong argument why higher-order operators have to be small. In the spirit that a useful dual description should not be too complicated, we simply investigate in this paper to what extent the effective action (42) is compatible with observation. If successful, the neglected subleading terms may be considered later for increased quantitative accuracy. Furthermore, we know that QCD contains higher resonances like the $\Delta$ or the axial-vector mesons. They are not described by the effective action (42). One may include them by the introduction of additional fields for bound states. This is, however, not the purpose of the present paper. We only mention here that "integrating out" the missing resonances with lowest mass presumably gives the leading contribution to the neglected higher-order operators. Those will typically contain "nonlocal behavior" in the momentum range characteristic for the resonances.

For high enough momenta (say $q^{2}>(2 \mathrm{GeV})^{2}$ ) our description should coincide with perturbative QCD. Obviously, in this momentum range $g(q)$ should coincide with the perturbative gauge coupling, providing for a "matching" of couplings. Furthermore, the (tree) exchange of the scalars induces an effective four-quark-interaction. In the perturbative QCD picture this matches with (part of) the 1PI-four-quark-interaction generated by "box diagrams" with gluon exchange.

The essential point of our picture of the QCD-vacuum concerns the location of the minimum of the effective potential. We will concentrate here on two directions in field space according to

$$
\begin{gathered}
\phi_{a b}=\bar{\sigma} \delta_{a b} \\
\chi_{i j, a b}=\frac{1}{\sqrt{24}} \bar{\chi} \lambda_{j i}^{z} \lambda_{a b}^{z}=\frac{1}{\sqrt{6}} \bar{\chi}\left(\delta_{i a} \delta_{j b}-\frac{1}{3} \delta_{i j} \delta_{a b}\right)
\end{gathered}
$$


with real fields $\bar{\sigma}, \bar{\chi}$. Our central assumption states that the minimum of $U(\bar{\sigma}, \bar{\chi})$ occurs for nonvanishing

$$
\bar{\sigma}=\sigma_{0}, \bar{\chi}^{2}=\chi_{0}^{2}
$$

In this context we emphasize that the source term (45) preserves the $S U(3)_{c}$ color symmetry. The minimum at $\bar{\chi}^{2}=\chi_{0}^{2}$ therefore corresponds to a whole orbit in the field space of $\chi_{i j, a b}$. This orbit obtains by applying $S U(3)_{c^{-}}$ transformations on the configuration (47). In consequence, our statement about the minimum of the effective potential is perfectly gauge invariant! In contrast, for nonzero quark masses the minimum in the direction of $\phi_{a b}$, i.e. $\phi_{a b}=\sigma_{0} \delta_{a b}$, is unique. As it stands, there exists a simple argument in QCD that the effective potential for color non singlet fields has its minimum at zero, i.e. $\chi_{0}=0$. Our investigation for $\chi_{0} \neq 0$ can therefore only hold in a gauge fixed version or after a suitable redefinition of $\chi$ by a multiplication with phase factors that makes it formally gauge invariant.

\section{Higgs picture}

For most of the discussion of this paper we will employ a gauge invariant description which only uses the assumption that the minimum of $U$ occurs for nonzero $\bar{\chi}$, without fixing a particular direction in the orbit of minima. Nevertheless, the most striking features of our picture of the QCD-vacuum become easily apparent if we fix for a moment the direction in field space. In this section we describe this "Higgs picture" where the vacuum is characterized by a fixed field

$$
\left\langle\chi_{i j, a b}\right\rangle=\frac{1}{\sqrt{24}} \chi_{0} \lambda_{j i}^{z} \lambda_{a b}^{z}=\frac{1}{\sqrt{6}} \chi_{0}\left(\delta_{i a} \delta_{j b}-\frac{1}{3} \delta_{i j} \delta_{a b}\right) .
$$

This expectation value is invariant under a combination of $S U(3)_{c}$ transformations and vectorlike flavor transformations. More precisely, the symmetry $S U(3)_{V}$ of the vacuum consists of those transformations where identical left and right flavor rotations and transposed color rotations are performed with opposite angles. With respect to the transposed color rotations the quarks behave as antitriplets. In consequence, under the combined transformation they transform as an octet plus a singlet, with all quantum numbers identical to the baryon octet/singlet. In particular, the electric charges, as given by the generator $Q=\frac{1}{2} \lambda_{3}+\frac{1}{2 \sqrt{3}} \lambda_{8}$ of $S U(3)_{V}$, are integer. We therefore identify the quark field with the lowest baryon octet and singlet - this is quark-baryon duality. Similarly, the gluons transform as an octet of vector-mesons, again 
with the standard charges for the $\rho, K^{*}$ and $\omega$ mesons. We therefore describe these vector mesons by the gluon field - this is gluon-meson duality.

Due to the Higgs mechanism all gluons acquire a mass. For equal quark masses conserved $S U(3)_{V}$ symmetry implies that all masses are equal. They should be identified with the average mass of the vector meson multiplet $\bar{M}_{\rho}^{2}=\frac{2}{3} M_{K^{*}}^{2}+\frac{1}{3} M_{\rho}^{2}=(850 \mathrm{MeV})^{2}$. One finds

$$
\bar{M}_{\rho}=g\left|\chi_{0}\right|=850 \mathrm{MeV} .
$$

The breaking of chiral symmetry by $\sigma_{0}$ and $\chi_{0}$ also induces masses for the baryon octet and singlet. The singlet mass is larger than the octet mass (see sect. 5).

In order to get familiar with the Higgs picture of QCD, it seems useful to understand in more detail the consequences of spontaneous color symmetry breaking for the electromagnetic interactions of hadrons. We start by adding to the effective Lagrangian (42) a coupling to a $U(1)$-gauge field $\tilde{B}_{\mu}$ by making derivatives covariant

$$
\begin{aligned}
D_{\mu} \psi & =\left(\partial_{\mu}-i \tilde{e} \tilde{Q} \tilde{B}_{\mu}\right) \psi \\
D_{\mu} \gamma_{i j} & =\partial_{\mu} \gamma_{i j}-i \tilde{e}\left[\tilde{Q}, \gamma_{i j}\right] \tilde{B}_{\mu}-\ldots
\end{aligned}
$$

with $\tilde{Q}=\frac{1}{2} \lambda_{3}+\frac{1}{2 \sqrt{3}} \lambda_{8}=\operatorname{diag}\left(\frac{2}{3},-\frac{1}{3},-\frac{1}{3}\right)$ acting on the flavor indices. Furthermore we supplement the Maxwell term

$$
\mathcal{L}_{\tilde{B}}=\frac{1}{4} \tilde{B}^{\mu \nu} \tilde{B}_{\mu \nu}, \tilde{B}_{\mu \nu}=\partial_{\mu} \tilde{B}_{\nu}-\partial_{\nu} \tilde{B}_{\mu}
$$

Whereas the quarks carry fractional $\tilde{Q}$, the abelian charges of the scalars are integer. In particular, the expectation value $\langle\phi\rangle$ is neutral, $[\tilde{Q},\langle\phi\rangle]=0$, whereas some components of $\left\langle\chi_{i j, a b}>\right.$ carry charge, namely for $k=2,3$

$$
\begin{aligned}
& {\left[\tilde{Q},<\chi_{1 k, 1 k}>\right]=<\chi_{1 k, 1 k}>} \\
& {\left[\tilde{Q},<\chi_{k 1, k 1}>\right]=-<\chi_{k 1, k 1}>}
\end{aligned}
$$

The expectation value (47) therefore also breaks the local $U(1)$ symmetry associated with $\tilde{Q}$. The abelian color charge $\left(Q_{C}\right)_{i j}=\frac{1}{2}\left(\lambda_{3}\right)_{i j}+\frac{1}{2 \sqrt{3}}\left(\lambda_{8}\right)_{i j}$ of these fields is, however, equal to $\tilde{Q}$. In consequence, a local abelian symmetry with generator $\tilde{Q}-Q_{C}$ remains unbroken

$$
\tilde{Q}_{a c}<\chi_{i j, c b}>-<\chi_{i j, a c}>\tilde{Q}_{c b}-\left(Q_{C}\right)_{i l}<\chi_{l j, a b}>+<\chi_{i l, a b}>\left(Q_{C}\right)_{l j}=0 .
$$

The corresponding gauge field corresponds to the photon. 
The situation encountered here is completely analogous to the Higgs mechanism in electroweak symmetry breaking. The mixing between the hypercharge boson and the $W_{3}$ boson in the electroweak theory appears here as a mixing between $\tilde{B}_{\mu}$ and a particular gluon field $\tilde{G}_{\mu}$ which corresponds to $A_{i j, \mu}=\frac{\sqrt{3}}{2}\left(Q_{C}\right)_{i j} \tilde{G}_{\mu}$. Let us restrict the discussion to the gauge bosons $\tilde{B}_{\mu}$ and $\tilde{G}_{\mu}$. Then the covariant derivative for fields with a fixed value of $\tilde{Q}$ and $Q_{C}$ is given by

$$
D_{\mu}=\partial_{\mu}-i \tilde{e} \tilde{B}_{\mu} \tilde{Q}-i \tilde{g} \tilde{G}_{\mu} Q_{C}
$$

with $\tilde{g}=\frac{\sqrt{3}}{2} g$. Due to the "charged" expectation values (53) a linear combination of $\tilde{B}_{\mu}$ and $\tilde{G}_{\mu}$ gets massive, as can be seen from the quadratic Lagrangian

$$
\mathcal{L}_{e m}^{(2)}=\frac{1}{4} \tilde{B}^{\mu \nu} \tilde{B}_{\mu \nu}+\frac{1}{4} \tilde{G}^{\mu \nu} \tilde{G}_{\mu \nu}+\frac{2}{3} \chi_{0}^{2}\left(\tilde{g} \tilde{G}^{\mu}+\tilde{e} \tilde{B}^{\mu}\right)\left(\tilde{g} \tilde{G}_{\mu}+\tilde{e} \tilde{B}_{\mu}\right) .
$$

The massive neutral vector meson $R_{\mu}$ and the massless photon $B_{\mu}$ are related to $\tilde{G}_{\mu}$ and $\tilde{B}_{\mu}$ by a mixing angle

$$
\begin{aligned}
R_{\mu} & =\cos \theta_{e m} \tilde{G}_{\mu}+\sin \theta_{e m} \tilde{B}_{\mu} \\
B_{\mu} & =\cos \theta_{e m} \tilde{B}_{\mu}-\sin \theta_{e m} \tilde{G}_{\mu} \\
t g \theta_{e m} & =\frac{\tilde{e}}{\tilde{g}}
\end{aligned}
$$

and we note that the mass of the neutral vector meson is somewhat enhanced by the mixing

$$
M_{V_{0}}=g \chi_{0} / \cos \theta_{e m} .
$$

The mixing is, however, tiny for the large value $\bar{\alpha}_{s}=g^{2} / 4 \pi \approx 3$ that we will find below. In terms of the mass eigenstates the covariant derivative (55) reads now

$$
D_{\mu}=\partial_{\mu}-i e Q B_{\mu}-i \tilde{g} \cos \theta_{e m}\left(Q_{C}+t g^{2} \theta_{e m} \tilde{Q}\right) R_{\mu}
$$

and we observe the universal electromagnetic coupling

$$
e=\tilde{e} \cos \theta_{e m}
$$

for all particles with electric charge $Q=\tilde{Q}-Q_{C}$. This coupling is exactly the same for the colored quarks and the colorless leptons as it should be for the neutrality of atoms. For an illustration we show the charges $Q_{C}, \tilde{Q}$ and $Q$ for the nine light quarks in table 1 . 


\begin{tabular}{c|ccc|c|} 
& $\tilde{Q}$ & $Q_{c}$ & $Q$ & \\
\hline$u_{1}$ & $2 / 3$ & $2 / 3$ & 0 & $\Sigma^{0}, \Lambda^{0}, S^{0}$ \\
$u_{2}$ & $2 / 3$ & $-1 / 3$ & 1 & $\Sigma^{+}$ \\
$u_{3}$ & $2 / 3$ & $-1 / 3$ & 1 & $p$ \\
\hline$d_{1}$ & $-1 / 3$ & $2 / 3$ & -1 & $\Sigma^{-}$ \\
$d_{2}$ & $-1 / 3$ & $-1 / 3$ & 0 & $\Sigma^{0}, \Lambda^{0}, S^{0}$ \\
$d_{3}$ & $-1 / 3$ & $-1 / 3$ & 0 & $n$ \\
\hline$s_{1}$ & $-1 / 3$ & $2 / 3$ & -1 & $\Xi^{-}$ \\
$s_{2}$ & $-1 / 3$ & $-1 / 3$ & 0 & $\Xi^{0}$ \\
$s_{3}$ & $-1 / 3$ & $-1 / 3$ & 0 & $\Lambda^{0}, S^{0}$
\end{tabular}

Table 1: Abelian charges of quarks and association with baryons. The baryon singlet is denoted by $S^{0}$.

The Higgs picture (with fixed direction of $\langle\chi>$ ) yields a particularly simple description of the mass generation for the gluons and the charges of the physical excitations. It has, however, some shortcomings concerning the simplicity of the description of the meson interactions and the correct interpretation of the global baryon number of the excitations [1]. These issues can be dealt with in a much easier way in the gauge invariant description.

\section{$5 \quad$ Nonlinear fields}

Let us now turn back to a gauge invariant description and use appropriate nonlinear fields. We will recover all the findings of the "Higgs picture" in the preceding section, now in a gauge invariant way. Indeed, we never need an assumption that an octet condensate occurs in a particular direction in field space (which would correspond to "spontaneous symmetry breaking" of the color symmetry). Furthermore, the interactions of the light mesons are most easily described in the gauge-invariant picture, using nonlinear fields ${ }^{9}$. For the "Goldstone directions" we introduce unitary matrices $W_{L}, W_{R}, v$ and

\footnotetext{
${ }^{9}$ On the level of the effective action the quantum fluctuations are already included. Therefore Jacobians for nonlinear field transformations play no role. All "coordinate choices" in the space of fields are equivalent.
} 
define

$$
\begin{aligned}
\psi_{L} & =Z_{\psi}^{-1 / 2} W_{L} N_{L} v, \psi_{R}=Z_{\psi}^{-1 / 2} W_{R} N_{R} v \\
\bar{\psi}_{L} & =Z_{\psi}^{-1 / 2} v^{\dagger} \bar{N}_{L} W_{L}^{\dagger}, \bar{\psi}_{R}=Z_{\psi}^{-1 / 2} v^{\dagger} \bar{N}_{R} W_{R}^{\dagger} \\
A_{\mu} & =-v^{T} V_{\mu}^{T} v^{*}-\frac{i}{g} \partial_{\mu} v^{T} v^{*}, U=W_{R} W_{L}^{\dagger} \\
\phi & =W_{R} S W_{L}^{\dagger}, \chi_{i j, a b}=\left(W_{R}\right)_{a c} v_{i k}^{T} X_{k l, c d} v_{l j}^{*}\left(W_{L}^{\dagger}\right)_{d b} .
\end{aligned}
$$

Here we have again extended the matrix notation to the color indices with the quark/baryon nonet represented by a complex $3 \times 3$ matrix $\psi \equiv \psi_{a i}, \bar{\psi} \equiv$ $\bar{\psi}_{i a}$. The decomposition of $\phi$ is such that $S$ is a hermitean matrix. The restrictions on $X$, which are necessary to avoid double counting, play no role in the present work. Besides $v$ all nonlinear fields are color singlets. The field $v^{\dagger}$ transforms as a color anti-triplet similar to a quark-quark pair or diquark. By virtue of triality - the center elements of global $S U(3)_{c}$ and $S U(3)_{L} \times S U(3)_{R}$ are identified with particular global transformations of the $U(1)_{B}$-symmetry which corresponds to conserved baryon number - the fields belonging to color representations in the class of the antitriplets carry baryon number $2 / 3(\bmod 1)$. We therefore assign to $v^{\dagger}$ (or the product $W^{\dagger} v^{\dagger}$ ) a nonvanishing baryon number $B=2 / 3$ (as appropriate for a diquark). In consequence, the baryons $N \sim W^{\dagger} \psi v^{\dagger}$ transform as color singlets with baryon number $B=1$, as it should be.

The nonlinear fields (61) are adapted to the physical excitations around the minimum of the effective potential. We encounter the gauge singlet baryon field $N$ and the vector-meson fields $V_{\mu}$. The pseudoscalar mesons correspond to the (pseudo-) Goldstone bosons described by $U=W_{R} W_{L}^{\dagger}$. We will see below that the remaining nonlinear degrees of freedom contained in $v$ and $W_{L, R}$ are gauge degrees of freedom. The effective action (42) can now be expressed in terms of the physical fields $N, V^{\mu}, U$ by inserting the definitions (61). In this form it will be straightforward to extract the physical excitations and their interactions.

Before proceeding with this program, we may verify that the effective action is invariant under the following field transformations

$$
\begin{aligned}
& \delta W_{L}=i \Theta_{L} W_{L}-i W_{L} \Theta_{P}, \delta W_{R}=i \Theta_{R} W_{R}-i W_{R} \Theta_{P} \\
& \delta v=i \Theta_{P} v+i v \Theta_{C}^{T}, \delta U=i \Theta_{R} U-i U \Theta_{L} \\
& \delta N_{L}=i\left[\Theta_{P}, N_{L}\right], \delta N_{R}=i\left[\Theta_{P}, N_{R}\right], \delta V_{\mu}=i\left[\Theta_{P}, V_{\mu}\right]+\frac{1}{g} \partial_{\mu} \Theta_{P} \text {, } \\
& \delta S=i\left[\Theta_{P}, S\right],(\delta X)_{i j, a b}=i\left(\Theta_{P}\right)_{a c} X_{i j, c b}-i X_{i j, a c}\left(\Theta_{P}\right)_{c b} \\
& -i\left(\Theta_{P}^{T}\right)_{i k} X_{k j, a b}+i X_{i k, a b}\left(\Theta_{P}^{T}\right)_{k j} \text {. }
\end{aligned}
$$


Here $\Theta_{c}, \Theta_{L}, \Theta_{R}$ are special unitary $3 \times 3$ matrices corresponding to $S U(3)_{c}, S U(3)_{L}$ and $S U(3)_{R}$, respectively. (We have no displayed additional global symmetries like the one corresponding to baryon number.) As a consequence of the nonlinear formulation one observes the appearance of a new local symmetry $U(3)_{P}=S U(3)_{P} \times U(1)_{P}$ under which the nucleons $N$ and the vector meson fields $V_{\mu}$ transform as octets and singlets

$$
\Theta_{P}=\frac{1}{2}\left(\theta_{P}^{z}(x) \lambda_{z}+\theta_{P}^{0}(x) \lambda_{0}\right), \lambda_{0} \equiv \frac{2}{\sqrt{6}} .
$$

This symmetry acts only on the nonlinear fields whereas $\psi, A_{\mu}, \phi$ and $\chi$ are invariant. It reflects the possibility of local reparameterizations of the nonlinear fields. It will play the role of the hidden gauge symmetry underlying vector dominance.

For the present investigation we omit the scalar excitations except for the "Goldstone directions" contained in $W_{L}, W_{R}, v$. We can therefore replace $X_{k l, a b}$ by the expectation value (47) and use $\langle S\rangle=\sigma_{0}$ such that

$$
\Phi=\sigma_{0} U \quad, \quad \chi_{i j, a b}=\frac{1}{\sqrt{6}} \chi_{0}\left\{\left(W_{R} v\right)_{a i}\left(v^{\dagger} W_{L}^{\dagger}\right)_{j b}-\frac{1}{3} U_{a b} \delta_{i j}\right\} .
$$

In terms of the nonlinear field coordinates the Lagrangian (42) reads

$$
\begin{aligned}
\mathcal{L}= & \operatorname{Tr}\left\{i \bar{N} \gamma^{\mu}\left(\partial_{\mu}+i v_{\mu}-i \gamma^{5} a_{\mu}\right) N-g \bar{N} \gamma^{\mu} N V_{\mu}\right\} \\
& +\frac{1}{2} \operatorname{Tr}\left\{V^{\mu \nu} V_{\mu \nu}\right\}+g^{2} \chi_{0}^{2} \operatorname{Tr}\left\{\tilde{V}^{\mu} \tilde{V}_{\mu}\right\}+\left(\frac{2}{9} \nu^{\prime} \chi_{0}^{2} \sigma_{0}-\nu \sigma_{0}^{3}\right) \cos \theta \\
& +\left(h \sigma_{0}-\frac{\tilde{h}}{3 \sqrt{6}} \chi_{0}\right) \operatorname{Tr}\left\{\bar{N} \gamma_{5} N\right\}+\frac{\tilde{h}}{\sqrt{6}} \chi_{0} \operatorname{Tr} \bar{N} \gamma^{5} \operatorname{Tr} N \\
& +\left(\sigma_{0}^{2}+\frac{7}{36} \chi_{0}^{2}\right) \operatorname{Tr}\left\{\partial^{\mu} U^{\dagger} \partial_{\mu} U\right\}+\frac{1}{12} \chi_{0}^{2} \partial^{\mu} \theta \partial_{\mu} \theta \\
& +\chi_{0}^{2} \operatorname{Tr}\left\{\tilde{v}^{\mu} \tilde{v}_{\mu}\right\}+2 g \chi_{0}^{2} \operatorname{Tr}\left\{\tilde{V}^{\mu} \tilde{v}_{\mu}\right\}
\end{aligned}
$$

with

$$
\begin{aligned}
& v_{\mu}=-\frac{i}{2}\left(W_{L}^{\dagger} \partial_{\mu} W_{L}+W_{R}^{\dagger} \partial_{\mu} W_{R}\right), \\
& a_{\mu}=\frac{i}{2}\left(W_{L}^{\dagger} \partial_{\mu} W_{L}-W_{R}^{\dagger} \partial_{\mu} W_{R}\right)=-\frac{i}{2} W_{R}^{\dagger} \partial_{\mu} U W_{L}, \\
& \operatorname{Tr} v_{\mu}=-\frac{i}{2} \partial_{\mu}\left(\ln \operatorname{det} W_{L}+\ln \operatorname{det} W_{R}\right), \\
& \operatorname{Tr} a_{\mu}=-\frac{i}{2} \operatorname{Tr} U^{\dagger} \partial_{\mu} U=-\frac{1}{2} \partial_{\mu} \theta, \\
& V_{\mu \nu}=\partial_{\mu} V_{\nu}-\partial_{\nu} V_{\mu}-i g\left[V_{\mu}, V_{\nu}\right]=\partial_{\mu} \tilde{V}_{\nu}-\partial_{\nu} \tilde{V}_{\mu}-i g\left[\tilde{V}_{\mu}, \tilde{V}_{\nu}\right], \\
& \tilde{V}_{\mu}=V_{\mu}-\frac{1}{3} \operatorname{Tr} V_{\mu}, \tilde{v}_{\mu}=v_{\mu}-\frac{1}{3} \operatorname{Tr} v_{\mu} .
\end{aligned}
$$


As it should be, $\mathcal{L}$ does not depend on $v$ and is therefore invariant under $S U(3)_{C}$ transformations. For a check of local $U(3)_{P}$ invariance we note

$$
\delta v_{\mu}=i\left[\Theta_{P}, v_{\mu}\right]-\partial_{\mu} \Theta_{P}, \delta a_{\mu}=i\left[\Theta_{P}, a_{\mu}\right]
$$

such that $v_{\mu}$ transforms the same as $-g V_{\mu}$ and $v_{\mu}+g V_{\mu}$ transforms homogeneously. One observes that the mass term for $\tilde{V}_{\mu}$ appears in the $U(3)_{P^{-}}$ invariant combination $\chi_{0}^{2} \operatorname{Tr}\left\{\left(\tilde{v}^{\mu}+g \tilde{V}^{\mu}\right)\left(\tilde{v}_{\mu}+g \tilde{V}_{\mu}\right)\right\}$. The nonlinear field $U$ only appears in derivative terms except for the phase $\theta$ associated to the $\eta^{\prime}$-meson. We associate $U$ in the standard way with the pseudoscalar octet of Goldstone bosons $\Pi^{z}$

$$
U=\exp \left(-\frac{i}{3} \theta\right) \exp \left(i \frac{\Pi^{z} \lambda_{z}}{f}\right)=\exp \left(-\frac{i}{3} \theta\right) \tilde{U}
$$

where the decay constant $f$ will be specified below.

The Lagrangian (65) contains mass terms and kinetic terms for an octet and a singlet of baryons. The same terms appear in the Higgs picture of sect. 4. We want to use the physical baryon masses in order to fix the Yukawa couplings $h$ and $\tilde{h}$. For this purpose we need to identify the baryon singlet. We will argue that $\Lambda(1405)$, which has the opposite parity as the nucleon or the lowest mass $\Lambda$-baryon, is an interesting candidate. This requires that the ratio $\tilde{h} \chi_{0} / h \sigma_{0}$ is negative. We observe that $h$ and $\tilde{h}$ can be chosen (via the form of the Hubbard-Stratonovich transformation) to be real and positive. Furthermore, we can use conventions where the current quark masses $m_{q}$ are real and a parity conserving ground state corresponds to positive $\sigma_{0}$. In the Higgs picture $C$ and $P$ are conserved by real $\chi_{0}$. Similarly, this holds for the expectation value of the gauge singlet $X_{k l, c d}$ (61). However, the sign of $\chi_{0}$ is not fixed and on the level of quarks we have no free phases left which could be used to make $\chi_{0}$ always positive. The relative sign between the singlet and octet contributions to the baryon masses $h \phi$ and $\tilde{h} \chi_{0}$ is therefore observable. (Note that no $S U(3)_{c}$-gauge transformation exists which reverses the sign of $\left.\chi_{i j, a b}.\right)$ Consider now the case of a negative singlet mass term $h \sigma_{0}+8 \tilde{h} \chi_{0} / 3 \sqrt{6}$, i.e. negative $\chi_{0}$. On the level of the nonlinear baryon fields we may apply a chiral phase transformation acting only on the singlet field $N_{1}=\operatorname{Tr} N / \sqrt{3}$ while leaving the octet field invariant. Choosing $\tilde{N}_{1 L}=i N_{1 L}, \tilde{N}_{1 R}=-i N_{1 R}$ we can render the singlet mass positive, but the parity of the singlet is now found opposite to the octet

$$
P: \tilde{N}_{1 L} \rightarrow \tilde{N}_{1 R}, \tilde{N}_{1 R} \rightarrow-\tilde{N}_{1 L} \quad, \quad N_{8 L} \rightarrow-N_{8 R}, N_{8 R} \rightarrow N_{8 L} .
$$

(In contrast, the $C$-parity is the same for the singlet and octet.) Quite interestingly, there exists a singlet state $\Lambda(1405)$ with the opposite parity to 
the $\Lambda$ in the baryon octet! (A singlet is not allowed in the ground state within the nonrelativistic quark model.) We will associate the $\Lambda(1405)$ baryon with the baryon singlet $S^{0}$

$$
S^{0} \equiv \tilde{N}_{1}=i \gamma^{5} N_{1}=\frac{i}{\sqrt{3}} \gamma^{5} \operatorname{Tr} N
$$

From eq. (65) one can now directly read the average (positive) masses of the baryon octet and singlet

$$
M_{8}=h \sigma_{0}-\frac{\tilde{h}}{3 \sqrt{6}} \chi_{0}=1.15 \mathrm{GeV}, M_{1}=-\left(h \sigma_{0}+\frac{8}{3} \frac{\tilde{h}}{\sqrt{6}} \chi_{0}\right)=1.4 \mathrm{GeV}
$$

The singlet-octet mass splitting is proportional to the octet condensate $\chi_{0}$

$$
M_{1}+M_{8}=-\frac{3}{\sqrt{6}} \tilde{h} \chi_{0}=2.55 \mathrm{GeV}
$$

With eq. (50) this determines the ratio

$$
\frac{\tilde{h}}{g}=2.45
$$

Similarly, one finds

$$
h \sigma_{0}=\frac{1}{9}\left(8 M_{8}-M_{1}\right)=866 \mathrm{MeV} .
$$

\section{Mesons and their interactions}

Before extracting the couplings between the physical mesons and the baryons we have to understand the role of the local reparameterization transformations $U(3)_{P}=S U(3)_{P} \times U(1)_{P}$. First of all, we note that $\operatorname{Tr} v_{\mu}$ can be eliminated by $U(1)_{P}$-gauge transformations and is therefore a pure gauge degree of freedom. Similarly, $\operatorname{Tr} V_{\mu}=-\frac{i}{g} \partial_{\mu} \ln$ det $v$ is also a gauge degree of freedom [1]. This explains why $\operatorname{Tr} v_{\mu}$ and $\operatorname{Tr} V_{\mu}$ only appear in the term bilinear in the nucleon fields. The vector fields $\tilde{V}_{\mu}$ are the gauge bosons of $S U(3)_{P}$. The nucleons transform as an octet and a singlet under $S U(3)_{P}$ and the same holds for the bilinear $a_{\mu}$. The fields contained in $W_{L}, W_{R}$ are antitriplets and $U$ is a singlet.

For a discussion of the mesons we have to eliminate the redundance of the local $U(3)_{P}$-transformations. We will employ the gauge choice

$$
W_{L}^{\dagger}=W_{R}=\xi
$$


which implies

$$
\begin{aligned}
& U=\xi^{2}, v_{\mu}=-\frac{i}{2}\left(\xi^{\dagger} \partial_{\mu} \xi+\xi \partial_{\mu} \xi^{\dagger}\right) \\
& a_{\mu}=-\frac{i}{2}\left(\xi^{\dagger} \partial_{\mu} \xi-\xi \partial_{\mu} \xi^{\dagger}\right)=-\frac{i}{2} \xi^{\dagger} \partial_{\mu} U \xi^{\dagger} \\
& \operatorname{Tr} v_{\mu}=0, \quad \operatorname{Tr} V_{\mu}=0 \\
& \phi=\sigma_{0} U, \xi_{i j, a b}=\frac{1}{\sqrt{6}} \chi_{0}\left(\xi_{a i} \xi_{j b}-\frac{1}{3} U_{a b} \delta_{i j}\right) \\
& \left(D_{\mu} \chi\right)_{i j, a b}=\frac{1}{\sqrt{6}} \chi_{0}\left[\partial_{\mu}\left(\xi_{a i} \xi_{j b}-\frac{1}{3} U_{a b} \delta_{i j}\right)+i g\left(\left(\xi \tilde{V}_{\mu}\right)_{a i} \xi_{j b}-\xi_{a i}\left(\tilde{V}_{\mu} \xi\right)_{j b}\right)\right]
\end{aligned}
$$

This gauge is singled out by the fact that $v_{\mu}$ contains no term linear in $\Pi^{z}$ or $\theta[1]$

$$
v_{\mu}=\tilde{v}_{\mu}=-\frac{i}{2 f^{2}}\left[\Pi, \partial_{\mu} \Pi\right]=-\frac{i}{8 f^{2}}\left[\lambda_{y}, \lambda_{z}\right] \Pi^{y} \partial_{\mu} \Pi^{z}+\ldots
$$

where we have chosen the parameterization

$$
\begin{aligned}
& \xi=\tilde{\xi} \exp \left(-\frac{i}{6} \theta\right), \operatorname{det} \tilde{\xi}=1 \\
& \tilde{\xi}=\exp \left(\frac{i}{2 f} \Pi^{z} \lambda_{z}\right)=\exp \left(\frac{i}{f} \Pi\right) .
\end{aligned}
$$

As for any gauge where $v_{\mu}$ contains no term linear in $\Pi$ or $\theta$, the term $\sim \operatorname{Tr} \tilde{v}^{\mu} \tilde{V}_{\mu}$ contains only interactions and does not affect the propagators. We can therefore associate $\Pi^{z}$ with the physical pions. Also the term $\operatorname{Tr} \tilde{v}^{\mu} \tilde{v}_{\mu}$ involves at least four meson fields and is irrelevant for the propagator of the pseudoscalar mesons.

The bilinear $a_{\mu}$ reads in the gauge (75)

$$
a_{\mu}=\frac{1}{2 f} \lambda_{z} \partial_{\mu} \Pi^{z}-\frac{1}{6} \partial_{\mu} \theta .+O\left(\Pi^{3}, . .\right)
$$

The effective action (65) contains therefore a cubic coupling between two baryons and a pion. As it should be for pseudo-Goldstone bosons, this is a derivative coupling. For the gauge (75) one has a simple realisation of the 
discrete transformations $P$ and $C$ on the level of nonlinear fields ${ }^{10}$

$$
\begin{aligned}
P: & N_{L} \rightarrow-N_{R}, N_{R} \rightarrow N_{L}, \\
& W_{L} \rightarrow W_{R}, W_{R} \rightarrow W_{L}, v \rightarrow v, U \rightarrow U^{\dagger}, \xi \rightarrow \xi^{\dagger} \\
& v_{\mu} \rightarrow v_{\mu}, a_{\mu} \rightarrow-a_{\mu}, V_{\mu} \rightarrow V_{\mu} \\
& \\
C: \quad & N_{L} \rightarrow c \bar{N}_{R}^{T}, N_{R} \rightarrow-c \bar{N}_{L}^{T}, \\
& W_{L} \rightarrow W_{R}^{*}, W_{R} \rightarrow W_{L}^{*}, v \rightarrow v^{*}, U \rightarrow U^{T}, \xi \rightarrow \xi^{T} \\
& v_{\mu} \rightarrow-v_{\mu}^{T}, a_{\mu} \rightarrow a_{\mu}^{T}, V_{\mu} \rightarrow-V_{\mu}^{T} .
\end{aligned}
$$

The gauge condition (75) can also be written as $W_{L} W_{R}=W_{R} W_{L}=1$ and is manifestly invariant under $P$ and $C$. The coupling $\operatorname{Tr} \bar{N} \gamma^{\mu} \gamma^{5} a_{\mu} N$ is a standard coupling of baryons to the axial vector current.

The kinetic term for the pseudoscalar mesons can be inferred from

$$
\begin{aligned}
\mathcal{L}_{\text {kin }}^{(P)} & =\left(\sigma_{0}^{2}+\frac{7}{36} \chi_{0}^{2}\right) \operatorname{Tr}\left\{\partial^{\mu} U^{\dagger} \partial_{\mu} U\right\}+\frac{1}{12} \chi_{0}^{2} \partial^{\mu} \theta \partial_{\mu} \theta \\
& =\frac{2}{f^{2}}\left(\sigma_{0}^{2}+\frac{7}{36} \chi_{0}^{2}\right) \partial^{\mu} \Pi^{z} \partial_{\mu} \Pi_{z}+\frac{1}{3}\left(\sigma_{0}^{2}+\frac{4}{9} \chi_{0}^{2}\right) \partial^{\mu} \theta \partial_{\mu} \theta
\end{aligned}
$$

Its standard normalization fixes a combination of $\sigma_{0}$ and $\chi_{0}$ in terms of the pseudoscalar decay constant $f$ which corresponds to an average in the octet [16] $f=\frac{2}{3} f_{K}+\frac{1}{3} f_{\pi}=106 \mathrm{MeV}$. For an optimal quantitative estimate of the expectation values $\sigma_{0}, \chi_{0}$ the so called "partial Higgs effect" should be included: the mixing between the pseudoscalars contained in $U(\sim i \bar{\psi} \psi)$ and those in the divergence of the axial-vector current $\left(\sim \partial_{\mu}\left(\bar{\psi} \gamma^{\mu} \gamma^{5} \psi\right)\right)$ introduces an additional negative contribution (partial Higgs effect)

$$
\Delta \mathcal{L}_{k i n}^{(P)}=-\frac{\Delta_{f}^{2}}{4} \operatorname{Tr}\left\{\partial^{\mu} \tilde{U}^{\dagger} \partial_{\mu} \tilde{U}\right\}-\Delta_{\theta}^{2} \partial^{\mu} \theta \partial_{\mu} \theta
$$

One infers a standard renormalization of the kinetic term for

$$
f^{2}=4 \sigma_{0}^{2}+\frac{7}{9} \chi_{0}^{2}-\Delta_{f}^{2}=\kappa_{f}^{2}\left(4 \sigma_{0}^{2}+\frac{7}{9} \chi^{2}\right)
$$

with

$$
\kappa_{f}=\left(1-\frac{\Delta_{f}^{2}}{4}\left(\sigma_{0}^{2}+\frac{7}{36} \chi_{0}^{2}\right)^{-1}\right)^{1 / 2}=\left(1+\frac{\Delta_{f}^{2}}{f^{2}}\right)^{-1 / 2} \leq 1 .
$$

The deviation of $\kappa_{f}$ from one is one of the most important effects of effective interactions not included in eq. (42). The effective interactions responsible

\footnotetext{
${ }^{10}$ Note that in our matrix notation $C$ acts as $\psi_{L} \rightarrow c \bar{\psi}_{R}^{T}, \psi_{R} \rightarrow-c \bar{\psi}_{L}^{T}$.
} 
for $\Delta_{f}^{2}$ also induce $S U(3)$-violating contributions to the pseudoscalar wave function renormalizations related to the strange quark mass ${ }^{11}$. This has led to a phenomenological estimate [16]

$$
\Delta_{f}^{2} \approx 0.45 f^{2}, \kappa_{f} \approx 0.83
$$

The pseudoscalar decay constants provide now for a quantitative estimate of the expectation values $\sigma_{0}$ and $\chi_{0}$

$$
f_{0}=2 \sqrt{\sigma_{0}^{2}+\frac{7}{36} \chi_{0}^{2}}=f / \kappa_{f}=\left(\frac{2}{3} f_{K}+\frac{1}{3} f_{\pi}\right) / \kappa_{f}=128 \mathrm{MeV} .
$$

In the approximation (42) one should use the leading order relation $f=f_{0}$, keeping in mind that nonleading effects will lower $f$ to its physical value.

In the gauge (75) the nonlinear Lagrangian (65) can now be written in terms of the normalized physical fields $N, \tilde{V}_{\mu}, \Pi$ and $\eta^{\prime}$ as

$$
\mathcal{L}=\mathcal{L}_{N}+\mathcal{L}_{U}+\mathcal{L}_{V}
$$

The part involving baryons and the axial interactions with the pseudoscalars reads

$$
\begin{aligned}
\mathcal{L}_{N}= & i \operatorname{Tr}\left\{\bar{N}_{8} \gamma^{\mu}\left(\partial_{\mu}-i \gamma^{5} \tilde{a}_{\mu}+\frac{i}{6 H_{\eta^{\prime}}} \gamma^{5} \partial_{\mu} \eta^{\prime}\right) N_{8}\right\}+M_{8} \operatorname{Tr}\left\{\bar{N}_{8} \gamma^{5} N_{8}\right\} \\
& +i \bar{N}_{1} \gamma^{\mu}\left(\partial_{\mu}+\frac{i}{6 H_{\eta^{\prime}}} \gamma^{5} \partial_{\mu} \eta^{\prime}\right) N_{1}-M_{1} \bar{N}_{1} \gamma^{5} N_{1} \\
& +\frac{1}{\sqrt{3}} \operatorname{Tr}\left\{\bar{N}_{8} \gamma^{\mu} \gamma^{5} \tilde{a}_{\mu}\right\} N_{1}+\frac{1}{\sqrt{3}} \bar{N}_{1} \gamma^{\mu} \gamma^{5} \operatorname{Tr}\left\{\tilde{a}_{\mu} N_{8}\right\} .
\end{aligned}
$$

Here we use the octet and singlet baryon fields (recall $N_{1}=-i \gamma^{5} S^{0}, \bar{N}_{1}=$ $\left.-i \bar{S}_{0} \gamma^{5}\right)$

$$
N_{1}=\frac{1}{\sqrt{3}} \operatorname{Tr} N, N_{8}=N-\frac{1}{3} \operatorname{Tr} N=N-\frac{1}{\sqrt{3}} N_{1}, \operatorname{Tr} N_{8}=0 .
$$

The interaction with the pseudoscalars involves the axial vector current

$$
\begin{aligned}
& \tilde{a}_{\mu}=a_{\mu}-\frac{1}{3} \operatorname{Tr} a_{\mu}=\frac{1}{2 f} \lambda_{z} \partial_{\mu} \Pi^{z}+O\left(\Pi^{3}\right), \operatorname{Tr} \tilde{a}_{\mu}=0, \\
& a_{\mu}=\tilde{a}_{\mu}-\frac{1}{6} \partial_{\mu} \theta=\tilde{a}_{\mu}-\frac{1}{6 H_{\eta^{\prime}}} \eta^{\prime} \quad, \quad \theta=\frac{1}{H_{\eta^{\prime}}} \eta^{\prime},
\end{aligned}
$$

\footnotetext{
${ }^{11}$ In the notation of ref. [16] one has $\Delta_{f}^{2} / f^{2}=-X_{\phi}^{-} \bar{\sigma}_{0}^{2} Z_{m}^{-1}$.
} 
whereas the interaction with the vector current will be contained in $\mathcal{L}_{V}$. The kinetic term and interactions for the pseudoscalars take the familiar form

$$
\mathcal{L}_{U}=\frac{f^{2}}{4} \operatorname{Tr}\left\{\partial^{\mu} \tilde{U}^{\dagger} \partial_{\mu} \tilde{U}\right\}+\frac{1}{2} \partial^{\mu} \eta^{\prime} \partial_{\mu} \eta^{\prime}-M_{\eta^{\prime}}^{2} H_{\eta^{\prime}}^{2} \cos \left(\eta^{\prime} / H_{\eta^{\prime}}\right)
$$

with

$$
\tilde{U}=\tilde{\xi}^{2}=\exp \left(i \frac{\Pi^{z} \lambda_{z}}{f}\right), \operatorname{det} \tilde{U}=1 .
$$

Finally, the terms involving vector mesons and vector currents are grouped in

$$
\begin{aligned}
\mathcal{L}_{V}= & \frac{1}{2} \operatorname{Tr}\left\{\tilde{V}^{\mu \nu} \tilde{V}_{\mu \nu}\right\}+\bar{M}_{\rho}^{2} \operatorname{Tr}\left\{\tilde{V}^{\mu} \tilde{V}_{\mu}\right\}+\frac{2}{g} \bar{M}_{\rho}^{2} \operatorname{Tr}\left\{\tilde{V}^{\mu} \tilde{v}_{\mu}\right\}+\frac{1}{g^{2}} \bar{M}_{\rho}^{2} \operatorname{Tr}\left\{\tilde{v}^{\mu} \tilde{v}_{\mu}\right\} \\
& -g \operatorname{Tr}\left\{\bar{N}_{8} \gamma^{\mu} N_{8} \tilde{V}_{\mu}\right\}-\frac{g}{\sqrt{3}}\left(\operatorname{Tr}\left\{\bar{N}_{8} \tilde{V}_{\mu}\right\} \gamma^{\mu} N_{1}+\bar{N}_{1} \gamma^{\mu} \operatorname{Tr}\left\{\tilde{V}_{\mu} N_{8}\right\}\right) \\
& -\operatorname{Tr}\left\{\bar{N}_{8} \gamma^{\mu} \tilde{v}_{\mu} N_{8}\right\}-\frac{1}{\sqrt{3}}\left(\operatorname{Tr}\left\{\bar{N}_{8} \tilde{v}_{\mu}\right\} \gamma^{\mu} N_{1}+\bar{N}_{1} \gamma^{\mu} \operatorname{Tr}\left\{\tilde{v}_{\mu} N_{8}\right\}\right)
\end{aligned}
$$

In the effective action (87) we have replaced the free parameters by $M_{8}, M_{1}, \bar{M}_{\rho}, M_{\eta^{\prime}}, f$ and $g$. We note that the number of parameters is reduced to six since only one particular combination of $\nu$ and $\nu^{\prime}$ appears in $M_{\eta^{\prime}}$. At this stage of our investigation only the effective gauge coupling has not yet been determined. The proportionality constant $H_{\eta^{\prime}}$ appearing in the couplings of $\eta^{\prime}$ reads [1]

$$
H_{\eta^{\prime}}=\left(\frac{1+\frac{16}{7} x}{1+x}\right)^{1 / 2} \frac{\kappa_{\theta}}{\kappa_{f}} \frac{\left(2 f_{K}+f_{\pi}\right)}{3 \sqrt{6}}
$$

where $\kappa_{\theta} / \kappa_{f}$ accounts for the details of the "partial Higgs effect". It can be related to the decay width of $\eta^{\prime}$ into two photons [16].

The interactions between individual baryons and mesons can be extracted 
by inserting the explicit representations

$$
\begin{gathered}
\Pi=\frac{1}{2} \Pi^{z} \lambda_{z}=\frac{1}{2}\left(\begin{array}{ccc}
\pi^{0}+\frac{1}{\sqrt{3}} \eta, & \sqrt{2} \pi^{+}, & \sqrt{2} K^{+} \\
\sqrt{2} \pi^{-}, & -\pi^{0}+\frac{1}{\sqrt{3}} \eta & \sqrt{2} K^{0} \\
\sqrt{2} K^{-}, & \sqrt{2} \bar{K}^{0}, & -\frac{2}{\sqrt{3}} \eta
\end{array}\right) \\
\tilde{V}_{\mu}=\frac{1}{2} \tilde{V}_{\mu}^{z} \lambda_{z}=\frac{1}{2}\left(\begin{array}{ccc}
\rho_{\mu}^{0}+\frac{1}{\sqrt{3}} V_{\mu}^{8}, & \sqrt{2} \rho_{\mu}^{+}, & \sqrt{2} K_{\mu}^{*+} \\
\sqrt{2} \rho_{\mu}^{-}, & -\rho_{\mu}^{0}+\frac{1}{\sqrt{3}} V_{\mu}^{8} & \sqrt{2} K_{\mu}^{* 0} \\
\sqrt{2} K_{\mu}^{*-}, & \sqrt{2} \bar{K}_{\mu}^{* 0}, & -\frac{2}{\sqrt{3}} V_{\mu}^{8}
\end{array}\right) \\
N_{8}=\left(\begin{array}{ccc}
\frac{1}{\sqrt{2}} \Sigma^{0}+\frac{1}{\sqrt{6}} \Lambda^{0}, & \Sigma^{+}, & p \\
\Sigma^{-}, & -\frac{1}{\sqrt{2}} \Sigma^{0}+\frac{1}{\sqrt{6}} \Lambda^{0}, & n \\
\Xi^{-}, & \Xi^{0}, & -\frac{2}{\sqrt{6}} \Lambda^{0}
\end{array}\right) \\
\bar{N}_{8}=\left(\begin{array}{ccc}
\frac{1}{\sqrt{2}} \bar{\Sigma}^{0}+\frac{1}{\sqrt{6}} \bar{\Lambda}^{0} . & \bar{\Sigma}^{-}, & \bar{\Xi}^{-} \\
\Sigma^{+}, & -\frac{1}{\sqrt{2}} \bar{\Sigma}^{0}+\frac{1}{\sqrt{6}} \bar{\Lambda}^{0}, & \bar{\Xi}^{0} \\
\bar{p}, & \bar{n}, & -\frac{2}{\sqrt{6}} \bar{\Lambda}^{0}
\end{array}\right)
\end{gathered}
$$

It is obvious that the effective action (87) predicts a multitude of different interactions between the low mass hadrons. The electromagnetic interactions are incorporated by covariant derivatives.

Finally, the addition of the explicit chiral symmetry breaking by the current quark masses (45)

$$
\mathcal{L}_{j}=-\frac{1}{2} Z_{\phi}^{-1 / 2} a_{q} \sigma_{0} \operatorname{Tr}\left\{\bar{m}\left(U+U^{\dagger}\right)\right\}
$$

leads to mass terms for the pseudoscalars [17] and contributes to their interactions, in accordance with chiral perturbation theory (cf. eq. (25)).

\section{$7 \quad$ Electromagnetic interactions}

As a first probe of our picture we may use the electromagnetic interactions of mesons and baryons. The electromagnetic interactions of the mesons are all contained in the covariant kinetic term for the scalars

$$
\begin{aligned}
\left(D^{\mu} \gamma_{i j, a b}\right)^{*} D_{\mu} \gamma_{i j, a b}= & \chi_{0}^{2} \operatorname{Tr}\left\{\left(\hat{v}^{\mu}+g \tilde{V}^{\mu}-\tilde{e} \tilde{B}^{\mu} \tilde{Q}\right)\left(\hat{v}_{\mu}+g \tilde{V}_{\mu}-\tilde{e} \tilde{B}_{\mu} \tilde{Q}\right)\right\} \\
& +\frac{f^{2}}{4} \operatorname{Tr}\left\{\left(D^{\mu} U\right)^{\dagger} D_{\mu} U\right\}+\frac{1}{12} \chi_{0}^{2} \partial^{\mu} \theta \partial_{\mu} \theta
\end{aligned}
$$

with generator $\tilde{Q}=\operatorname{diag}(2 / 3,-1 / 3,-1 / 3)$ for the electric charge. Here $D_{\mu} U=\partial_{\mu} U-i \tilde{e} \tilde{B}_{\mu}[\tilde{Q}, U]$ and the covariant vector current reads (with 


$$
\begin{aligned}
\left.\tilde{D}_{\mu} \xi=\partial_{\mu} \xi-i \tilde{e} \tilde{B}_{\mu}[\tilde{Q}, \xi]\right) & \\
\hat{v}_{\mu} & =-\frac{i}{2}\left(W_{L}^{\dagger} \tilde{D}_{\mu} W_{L}+W_{R}^{\dagger} \tilde{D}_{\mu} W_{R}\right) \\
& =-\frac{i}{2}\left(\xi^{\dagger} \tilde{D}_{\mu} \xi+\xi \tilde{D}_{\mu} \xi^{\dagger}\right) \\
& =v_{\mu}-\frac{\tilde{e}}{2} \tilde{B}_{\mu}\left(\xi^{\dagger} \tilde{Q} \xi+\xi \tilde{Q} \xi^{\dagger}-2 \tilde{Q}\right) .
\end{aligned}
$$

Observing that both $\hat{v}_{\mu}$ and $g \tilde{V}_{\mu}-\tilde{e} \tilde{B}_{\mu} \tilde{Q}$ transform homogeneously with respect to the electromagnetic gauge transformations

$$
\delta_{e m} \hat{v}_{\mu}=i \beta\left[\tilde{Q}, \hat{v}_{\mu}\right], \delta_{e m}\left(g \tilde{V}_{\mu}-\tilde{e} \tilde{B}_{\mu} \tilde{Q}\right)=i \beta\left[Q,\left(g \tilde{V}_{\mu}-\tilde{e} \tilde{B}_{\mu} \tilde{Q}\right)\right]
$$

the gauge invariance of (97) can be easily checked. The particular combination of vector currents in (97) is dictated by the combination of electromagnetic gauge invariance and local reparameterization symmetry. Only this combination transforms homogeneously with respect to both $U(3)_{P}$ local reparameterizations and electromagnetic $U(1)$ gauge transformations.

The interactions (97) coincide with those of a "hidden local chiral symmetry" 18 if one replaces $\hat{v}_{\mu}$ by $v_{\mu}$. (The small difference between our result and the "hidden symmetry" approach is due to the somewhat different electromagnetic transformation properties of the nonlinear fields). If one restricts the discussion to $\rho$-mesons and pions, $\tilde{V}_{\mu}=\frac{1}{2} \vec{\rho}_{V \mu} \vec{\tau}, \Pi=\frac{1}{2} \vec{\pi} \vec{\tau}$, and neglects the difference between $\tilde{e}$ and $e$, one finds for the term involving the vector mesons and currents

$$
\begin{aligned}
\mathcal{L}_{V V}= & a f_{\pi}^{2} \operatorname{Tr}\left(\hat{v}_{\mu}+\frac{1}{2} g_{\rho} \vec{\rho}_{V \mu} \vec{\tau}-\frac{1}{2} e \tilde{B}_{\mu} \tau_{3}\right)^{2} \\
= & a f_{\pi}^{2} \operatorname{Tr}\left[\frac{1}{4 f_{\pi}^{2}}\left(\left(\vec{\pi} \times \partial_{\mu} \vec{\pi}\right) \vec{\tau}\right)+\frac{1}{2} g_{\rho}\left(\vec{\rho}_{V \mu} \vec{\tau}\right)-\frac{1}{2} e \tilde{B}_{\mu} \tau_{3}\right. \\
& \left.-\frac{e}{4 f_{\pi}^{2}} \tilde{B}_{\mu}\left(\pi_{3}(\vec{\pi} \vec{\tau})-(\vec{\pi} \vec{\pi}) \tau_{3}\right)\right]^{2}+\ldots
\end{aligned}
$$

For the second equality in eq. (100) we have only retained terms quadratic in $\pi$ in an expansion of $\hat{v}_{\mu}$ and we have replaced $f$ by $f_{\pi}$. The first expression in eq. (100) is actually more general than the result of the particular effective action (42) for which one has

$$
\begin{aligned}
g_{\rho} & =g, \\
a & =\frac{\chi_{0}^{2}}{f_{\pi}^{2}}=\frac{9}{7 \kappa_{f}^{2}} \frac{x}{1+x} \frac{f^{2}}{f_{\pi}^{2}} \approx 2.4 \frac{x}{1+x} .
\end{aligned}
$$


In consequence of the symmetries, additional interactions will change the values of a $a$ and $g_{\rho}$ without affecting the structure of the invariant. Such additional interactions will also generate new invariants involving higher derivatives [1].

The contributions in eq (100) with canonical dimension $\leq 4$ can be written in the form

$$
\begin{aligned}
\mathcal{L}_{V V}= & \frac{1}{2} M_{\rho}^{2} \vec{\rho}_{V}^{\mu} \vec{\rho}_{V \mu}-e g_{\rho \gamma} \rho_{V 3}^{\mu} \tilde{B}_{\mu}+\frac{1}{2} m_{B}^{2} \tilde{B}^{\mu} \tilde{B}_{\mu} \\
& +g_{\rho \pi \pi} \vec{\rho}_{V}^{\mu}\left(\vec{\pi} \times \partial_{\mu} \vec{\pi}\right)+g_{\gamma \pi \pi}^{(V)} \tilde{B}^{\mu}\left(\vec{\pi} \times \partial_{\mu} \vec{\pi}\right)_{3} \\
& +g_{\rho \gamma \pi \pi} \tilde{B}_{\mu}\left[(\vec{\pi} \vec{\pi}) \rho_{V 3}^{\mu}-\left(\vec{\rho}_{V}^{\mu} \vec{\pi}\right) \pi_{3}\right] \\
& -\frac{1}{2} a e^{2} \tilde{B}_{\mu} \tilde{B}^{\mu}\left[\left(\vec{\pi} \vec{\pi}-\pi_{3}^{2}\right]+\ldots\right.
\end{aligned}
$$

with

$$
\begin{aligned}
& M_{\rho}^{2}=a g_{\rho}^{2} f_{\pi}^{2}, g_{\rho \gamma}=a g_{\rho} f_{\pi}^{2}, g_{\rho \pi \pi}=\frac{1}{2} a g_{\rho} \\
& m_{B}^{2}=a e^{2} f_{\pi}^{2}, g_{\gamma \pi \pi}^{(V)}=-\frac{1}{2} a e, g_{\rho \gamma \pi \pi}=\frac{1}{2} a e g_{\rho} .
\end{aligned}
$$

The effective action (42) therefore leads to the very successful KSFR relation 19, 18

$$
g_{\rho \gamma}=2 f_{\pi}^{2} g_{\rho \pi \pi}
$$

which relates the decay $\rho \rightarrow 2 \pi$ (with $g_{\rho \pi \pi} \approx 6$, see next section) to the electromagnetic properties of the $\rho$-meson, in particular the decay $\rho_{0} \rightarrow e^{+} e^{-}$ (with $\Gamma\left(\rho_{0} \rightarrow e^{+} e^{-}\right)=6.62 \mathrm{keV}$ and $g_{\rho \gamma}=0.12 \mathrm{GeV}^{2}$ ).

The relation

$$
M_{\rho}^{2}=\frac{4}{a} g_{\rho \pi \pi}^{2} f_{\pi}^{2}
$$

requires $a=2.1$, implying

$$
\chi_{0}=135 \mathrm{MeV} \text {. }
$$

One could use eq. (101) for an estimate of the relative octet contribution to the pion decay constant and eq. (50) for an estimate of $g$

$$
x=7 \quad, \quad \sigma_{0}=22 \mathrm{MeV} \quad, \quad g=6.3 .
$$

This should, however, not be taken too literally in view of possible substantial $S U(3)_{V}$-violating effects from the nonzero strange quark mass. Also higher order operators may affect the relation (101). Furthermore, one should 
include the corrections from additional invariants. However, the possible additional invariants only affect the cubic and higher vertices, but not the mass terms. In particular, the relation

$$
g_{\rho \gamma}=g \chi_{0}^{2}=\frac{\bar{M}_{\rho}^{2}}{g}
$$

will only be modified by $S U(3)$-violating effects. It can be used for an independent estimate of $g$, yielding $g=6$. Because of the relative robustness of this last estimate we will use the observed value of $g_{\rho \gamma}$ as an input and assume $g=6$. All free parameters are then fixed by observation and the decay rate for $\rho \rightarrow 2 \pi$ is a first "prediction" of our model.

The electromagnetic $\gamma \pi \pi$ and $\gamma \gamma \pi \pi$ vertices also receive contributions from

$$
\begin{aligned}
\frac{1}{4} f_{\pi}^{2} \operatorname{Tr}\left\{D^{\mu} U^{\dagger} D_{\mu} U\right\}= & \frac{1}{2} \partial^{\mu} \vec{\pi} \partial_{\mu} \vec{\pi} \\
& +e \tilde{B}^{\mu}\left(\vec{\pi} \times \partial_{\mu} \vec{\pi}\right)_{3}+\frac{1}{2} e^{2} \tilde{B}_{\mu} \tilde{B}^{\mu}\left(\vec{\pi} \vec{\pi}-\pi_{3}^{2}\right)+\ldots
\end{aligned}
$$

One sees that for $a=2$ the two contributions (102) and (109) to the direct $\gamma \pi \pi$ vertex cancel. The electromagnetic interactions of the pions are then dominated by $\rho$-exchange (vector dominance), in agreement with observation. Otherwise stated, our model leads for the direct $\gamma \pi \pi$-coupling to the realistic relation

$$
g_{\gamma \pi \pi}=e\left(1-\frac{2 g_{\rho \pi \pi}^{2} f_{\pi}^{2}}{M_{\rho}^{2}}\right) .
$$

The vertex $\sim g_{\rho \gamma \pi \pi}$ contributes $^{12}$ to rare decays like $\rho_{0} \rightarrow \pi^{+} \pi^{-} \gamma$, with

$$
g_{\rho \gamma \pi \pi}=e g_{\rho \pi \pi} .
$$

We conclude that the electromagnetic interactions of the pseudoscalars as well as the vector mesons can be considered as a successful test of our simple model. The appearance of a local nonlinear reparameterization symmetry is a direct consequence of the "spontaneous breaking" of color. This symmetry, combined with the simple effective action (42), has led to the KSFR relation (104) and to vector dominance (110). At this stage the relations of the above discussion should be taken with a 20-30 percent uncertainty. In particular, the $S U(3)$-violation due to the nonzero strange quark mass needs to be dealt with more carefully. Nevertheless, the $\rho \rightarrow 2 \pi$ decay and the electromagnetic decays are all consistent and have allowed us a first determination of the size

\footnotetext{
${ }^{12}$ This vertex is absent in ref. [18].
} 
of the octet condensate $\chi_{0}$. The octet condensate is larger than the singlet condensate and dominates the pseudoscalar decay constant $f$.

Finally, the connection between the above discussion and the Higgs picture developed in sect. 4 is easily established if one realizes that the field

$$
\begin{aligned}
G_{\mu} & =-\sqrt{3} \operatorname{Tr}\left\{\tilde{Q} \tilde{V}_{\mu}\right\}=\sqrt{3} \operatorname{Tr}\left\{\tilde{Q}\left(v A_{\mu}^{T} v^{\dagger}+\frac{i}{g} \partial_{\mu} v v^{\dagger}\right)\right\} \\
& =-\left(\frac{\sqrt{3}}{2} \rho_{\mu}^{0}+\frac{1}{2} V_{\mu}^{8}\right)
\end{aligned}
$$

is the nonlinear correspondence of $\tilde{G}_{\mu}$ in sect. 4. With respect to the electromagnetic gauge transformations it transforms inhomogeneously

$$
\delta_{e m} G_{\mu}=-\frac{2}{\sqrt{3} g} \partial_{\mu} \beta=-\frac{1}{\tilde{g}} \partial_{\mu} \beta
$$

such that the linear combinations (57) $R_{\mu}=\cos \theta_{e m} G_{\mu}+\sin \theta_{e m} \tilde{B}_{\mu}, B_{\mu}=$ $\cos \theta_{e m} \tilde{B}_{\mu}-\sin \theta_{e m} G_{\mu}$ have the transformation properties of a heavy neutral boson and a photon

$$
\delta_{e m} B_{\mu}=\frac{1}{e} \partial_{\mu} \beta \quad, \quad \delta_{e m} R_{\mu}=0
$$

Inserting in $\mathcal{L}_{V}\left(\underline{93)} \tilde{V}_{\mu}=-\frac{\sqrt{3}}{2} G_{\mu} \tilde{Q}, \tilde{v}_{\mu}=0\right.$ and adding terms from covariant derivatives (151) involving $\tilde{B}_{\mu}($ cf. (100) $)$, we recover $\mathcal{L}_{e m}^{(2)}$ (56) with $\tilde{G}_{\mu}$ replaced by $G_{\mu}$. The (extended) electromagnetic interactions of the baryon octet $^{13}$ read

$$
\begin{aligned}
\mathcal{L}_{V N, 0}= & e B_{\mu} \operatorname{Tr}\left\{\bar{N}_{8} \gamma^{\mu}\left[\tilde{Q}, N_{8}\right]\right\} \\
& +\tilde{g} \cos \theta_{e m} R_{\mu} \operatorname{Tr}\left\{\bar{N}_{8} \gamma^{\mu}\left(N_{8} \tilde{Q}+t g^{2} \theta_{e m} \tilde{Q} N_{8}\right)\right\}
\end{aligned}
$$

One finds the standard coupling between the photon $B_{\mu}$ and the baryons according to their integer electric charge. More generally, we conclude from

$$
\begin{aligned}
& \tilde{e} \tilde{B}_{\mu}=e\left(B_{\mu}+\operatorname{tg} \theta_{e m} R_{\mu}\right) \\
& \tilde{g} G_{\mu}=\tilde{g} \cos \theta_{e m} R_{\mu}-e B_{\mu}
\end{aligned}
$$

that the charged leptons have a small direct coupling to a linear combination of the $\rho^{0}, \omega$ vector mesons corresponding to the term e $\operatorname{tg} \theta_{e m} R_{\mu}$ in $\tilde{e} \tilde{B}_{\mu}$. Similarly, the photon has a hadronic coupling from the term $-e B_{\mu}$ in $\tilde{g} G_{\mu}$. These mixing effects are governed by the coupling $g_{\gamma \rho}$ (104).

\footnotetext{
${ }^{13}$ We omit here terms involving the baryon singlet $N_{1}$ and take $\Pi=0$.
} 


\section{Vector mesons}

The vector mesons acquire a mass through the Higgs mechanism. They are also unstable due to the decay into two pseudoscalar mesons. Their interactions are contained in $\mathcal{L}_{V}$ (93). In particular, the cubic vertex between one vector meson and two pseudoscalars is

$$
\begin{aligned}
\mathcal{L}_{V \pi \pi} & =2 g \chi_{0}^{2} \operatorname{Tr}\left\{\tilde{V}^{\mu} \tilde{v}_{\mu}\right\}=-\frac{i g \chi_{0}^{2}}{f^{2}} \operatorname{Tr}\left\{\left[\Pi, \partial_{\mu} \Pi\right] \tilde{V}^{\mu}\right\} \\
& =-2 i g_{\rho \pi \pi} \operatorname{Tr}\left\{\left[\Pi, \partial_{\mu} \Pi\right] \tilde{V}^{\mu}\right\}
\end{aligned}
$$

with

$$
g_{\rho \pi \pi}=\frac{g}{2} \frac{\chi_{0}^{2}}{f^{2}}=\frac{M_{\rho}^{2}}{2 g f^{2}} .
$$

Considering only the effective action (42), one has $\kappa_{f}=1$ and would infer

$$
g_{\rho \pi \pi}=\frac{9 x}{14(1+x)} g \approx 4.6\left(\frac{x}{1+x}\right)^{1 / 2} .
$$

If we restrict these interactions to the $\rho$-mesons $\tilde{V}_{\mu}=\frac{1}{2} \vec{\rho}_{V \mu} \vec{\tau}$ and pions $\Pi=\frac{1}{2} \vec{\pi} \vec{\tau}, v_{\mu}=\frac{1}{4 f^{2}}\left(\vec{\pi} \times \partial_{\mu} \vec{\pi}\right) \vec{\tau}$, we obtain the familiar form

$$
\mathcal{L}_{\rho \pi \pi}=\frac{g \chi_{0}^{2}}{2 f^{2}} \epsilon_{i j k} \pi^{i} \partial_{\mu} \pi^{j} \rho_{V}^{k \mu}=g_{\rho \pi \pi}\left(\vec{\pi} \times \partial_{\mu} \vec{\pi}\right) \vec{\rho}_{V}^{\mu} .
$$

It is straightforward to compute the decay rate $\rho \rightarrow 2 \pi$ as

$$
\Gamma(\rho \rightarrow \pi \pi)=\frac{g_{\rho \pi \pi}^{2}}{48 \pi} \frac{\left(M_{\rho}^{2}-4 M_{\pi}^{2}\right)^{3 / 2}}{M_{\rho}^{2}} \simeq 150 \mathrm{MeV}
$$

and one infers the phenomenological value $g_{\rho \pi \pi} \simeq 6.0$. The discrepancy with eq. (119) reflects the difference between the realistic value $a \approx 2$ and a value of $a$ which would result from (101) for $\kappa_{f}=1, f_{\pi}=f$. The estimate (119) receives, however, important corrections. Omitted $S U(3)_{V}$-violating effects from the strange quark mass result in corrections $\sim 30 \%$. Furthermore, since eq. (101) is no symmetry relation, it is subject to modifications from the inclusion of additional fields. It is precisely the inclusion of these effects in eq. (101) (i.e. $f_{\pi} \neq f, \kappa_{f}<1$ ) that makes the prediction of our model for $g_{\rho \pi \pi}$ agree with observation.

The effective coupling between $\rho$-mesons and baryons (93) obeys

$$
\mathcal{L}_{\bar{N} N_{\rho}}=-\frac{g}{2} \operatorname{Tr}\left\{\bar{N}_{8} \gamma^{\mu} N_{8} \vec{\tau}\right\} \vec{\rho}_{V \mu}
$$


where we omit from now on electromagnetic effects. This implies that the effective action (42) does not contain a direct coupling of the $\rho$-mesons to protons and neutrons. The $\rho$-mesons only couple to strange baryons why the nucleon-nucleon interactions in the vector channel are mediated by the exchange of the $\omega$-meson. In the approximation of the effective action (42) possible contributions to the nucleon-nucleon interactions in the isospin triplet vector channel could only arise through two-pion interactions $\sim \operatorname{Tr}\left\{\bar{N}_{8} \gamma^{\mu} \tilde{v}_{\mu} N_{8}\right\}$

More generally, one may compute in our model the effective nucleonnucleon interactions and compare them with experimental information. The exchange of the mesons generates effective vertices for nucleon-nucleon scattering in the form of terms $\sim(\bar{N} N)^{2}$. For their determination one should solve the field equations for $\Pi$ and $\tilde{V}_{\mu}$ as functionals of the baryon fields $N_{8}$ in bilinear order $\sim \bar{N}_{8} N_{8}$. The solution has to be reinserted into the effective action. As a result of this procedure one finds nucleon-nucleon interactions in the pseudoscalar channel and in the isospin-singlet vector channel mediated by the exchange of $V_{8 \mu}$ but not in the isospin-triplet vector channel. (The- $\sigma$ exchange term in the scalar channel is also contained in our model once the non-Goldstone scalar excitations in $\phi$ and $\chi$ are included.) This absence of isospin-triplet vector channel nucleon-nucleon interactions seems not to be consistent with observation [20] but the size of the relevant coupling remains debated.

In summary, three shortcomings indicate that the effective action (42) gives only an insufficient picture of the hadronic interactions in the vector channel: (i) the absence of a physical $S U(3)$-singlet vector state (the ninth vector meson), (ii) the inaccurate estimate of the parameter $a$ in eq. (101) for $\kappa_{f}=1$, (iii) the absence of nucleon-nucleon interactions in the isospin-triplet vector channel. In addition, no axial-vector mesons are present. These shortcomings can be overcome once we include the effective four-quark interactions in the color singlet vector and axial-vector channel. The successful relations (104), (110) and (111) can be maintained, whereas the parameter $a$ and the relation between $M_{\rho}, g_{\rho \pi \pi}$ and $f_{\pi}$ will be modified. Mixing effects with the divergence of the axial vector induce the correction (82) and therefore $\kappa_{f}<1$. The effects from vector and axial-vector four-quark interactions are discussed in detail in [1]. Besides the "partial Higgs effect" (82) they account for the missing nucleon-nucleon interactions in the isospin triplet channel. They also contain the missing ninth vector meson, i.e. the $S U(3)_{C}$-singlet state, as well as the axial-vector mesons. Unfortunately, the additional effective interactions are parameterized by new unknown couplings.

At this point we also can make the connection to the formulation in sect. 2 which uses only gauge invariant composite operators. Indeed, we may in- 
troduce additional fields for the gauge singlet fermion bilinears transforming as a vector

$$
\rho_{L}^{\mu} \sim \bar{\psi}_{L} \gamma^{\mu} \psi_{L}, \rho_{R}^{\mu} \sim \bar{\psi}_{R} \gamma^{\mu} \psi_{R}
$$

Neglecting the axial vector degrees of freedom this will lead to an effective action $^{14} \Gamma\left[A_{\mu}, \varphi, \chi, \rho\right]$ which depends in addition on the fields

$$
\rho^{\mu}=\frac{1}{\sqrt{2}}\left(\rho_{L}^{\mu}+\rho_{R}^{\mu}\right)
$$

In presence of the "octet condensate" the fields $\rho^{\mu}$ and $A^{\mu}$ (more precisely $\rho^{\mu}$ and $V^{\mu}$ ) will mix and the low mass physical vector mesons correspond to an appropriate linear combination. One may reduce the degrees of freedom in the effective action by solving the field equations for one of the vector fields in presence of the other and reinserting the result into the effective action. After elimination of $\rho^{\mu}$ in favor of $A^{\mu}$ the effective action (42) may be viewed as the lowest order in a derivative expansion for the octet vector mesons. The missing couplings and the singlet vector meson are provided by $\rho^{\mu}$ [1]. In contrast, the formulation of sect. 2 in terms of gauge invariant vector fields is recovered if we eliminate $A_{\mu}$ and $\chi$ in favor of $\rho^{\mu}, \varphi$, i.e. $\Gamma\left[A_{\mu}, \varphi, \chi, \rho\right] \rightarrow \Gamma[\varphi, \rho]$. The result is then precisely the effective action for the gauge invariant bilinears similar to sect. 2. From there we can extract directly the gauge invariant correlation functions as "measured", for example, on the lattice.

More in detail, the mixing between $\rho^{\mu}$ and $A^{\mu}$ results from invariants of the type [1]

$$
\left(D_{\mu} \chi\right)_{i j a b}^{*} \chi_{i j a c}\left(\rho_{L}^{\mu}\right)_{c b}+\cdots \Rightarrow \chi_{0}^{2} \rho^{\mu} A_{\mu} .
$$

Expanding the effective action in terms quadratic in $A^{\mu}$ and $\rho^{\mu}$ one obtains

$$
\mathcal{L}_{\rho A}=\frac{1}{2} M^{2} \rho^{\mu} \rho_{\mu}+\frac{1}{2} A^{\mu}\left(-\partial^{2}+g^{2} \chi_{0}^{2}\right) A_{\mu}-\beta \chi_{0}^{2} \rho^{\mu} A_{\mu}+\ldots
$$

This leads to a lowest order field equation for $A^{\mu}$

$$
\left(-\partial^{2}+g^{2} \chi_{0}^{2}\right) A^{\mu}=\beta \chi_{0}^{2} \rho^{\mu} .
$$

Reinserting the solution into $\mathcal{L}_{\rho A}(126)$ we find the term quadratic in $\rho^{\mu}$

$$
\mathcal{L}_{\rho}=\frac{1}{2} \rho^{\mu} \frac{M^{2}}{-\partial^{2}+g^{2} \chi_{0}^{2}}\left(-\partial^{2}+g^{2} \chi_{0}^{2}-\beta^{2} \chi_{0}^{4} / M^{2}\right) \rho_{\mu} .
$$

\footnotetext{
${ }^{14}$ See ref. [1], appendix A for a detailed description of the structure of the effective action in presence of $\rho_{L}^{\mu}, \rho_{R}^{\mu}$, including a discussion of axial vectors.
} 
It is now straightforward to extract the $\rho$-meson mass from the field equation for $\rho^{\mu}$

$$
\left(-\partial^{2}+\bar{m}_{\rho}^{2}\right) \rho^{\mu}=0 .
$$

We see that the effective mass $\bar{m}_{\rho}$ is essentially the one found from the effective action (42), with a correction $\sim \beta^{2}$ due to the mixing effect

$$
\bar{m}_{\rho}^{2}=g^{2} \chi_{0}^{2}\left(1-\frac{\beta^{2} \chi_{0}^{2}}{g^{2} M^{2}}\right) .
$$

In summary, the effective action in presence of the scalar color octet field $\chi$ can also be considered as an intermediate step for the computation of the action for gauge singlet $\bar{q} q$ bilinears.

\section{Interactions of pseudoscalar mesons}

The cubic interactions between the pseudoscalar and the baryon octets are usually parameterized by

$$
\mathcal{L}_{N}^{(p)}=F \operatorname{Tr}\left\{\bar{N}_{8} \gamma^{\mu} \gamma^{5}\left[\tilde{a}_{\mu}, N_{8}\right]\right\}+D \operatorname{Tr}\left\{\bar{N}_{8} \gamma^{\mu} \gamma^{5}\left\{\tilde{a}_{\mu}, N_{8}\right\}\right\}
$$

Experimental values are $F=0.459 \pm 0.008$ and $D=0.798 \pm 0.008$. The effective action (42) leads to (cf. eq. (88) ) $F=D=0.5$. This can be considered as a good achievement since these couplings remain completely undetermined by the chiral symmetry. Also the reparameterization symmetry does not restrict the coupling constants $F$ and $D$. (This contrasts with the coupling of the vector current $\tilde{v}_{\mu}$.) In our context the relation $F=D=0.5$ is directly connected to the origin of these couplings from the quark kinetic term and therefore to quark-baryon duality. The partial Higgs effect provides for a correction $F+D=1+y_{N}$ and one infers $y_{N} \approx 0.26$ [1]. Contributions to $D-F \approx 0.34$ have to be generated from other higher-order invariants, as, for example, a momentum-dependent Yukawa coupling involving $D_{\mu} \chi$. We note that $D-F$ contributes to the $\eta$-nucleon coupling but not to the interaction between protons, neutrons and pions. The latter can be written in a more conventional form with the nucleon doublet $\mathcal{N}^{T}=(p, n)$ and $\tilde{a}_{\mu}$ restricted to a $2 \times 2$ matrix (by omitting the last line and column)

$$
\begin{aligned}
\mathcal{L}_{\mathcal{N}}^{(\pi)} & =g_{A} \overline{\mathcal{N}} \gamma^{\mu} \gamma^{5} \tilde{a}_{\mu} \mathcal{N} \\
g_{A} & =F+D
\end{aligned}
$$

The inclusion of weak interactions will replace the derivative in the definition (76) of $\tilde{a}_{\mu}$ by a covariant derivative involving a coupling to the $W$-boson [1]. The constant $g_{A}$ will therefore appear in the $\beta$-decay rate of the neutron. 
The vertex responsible for the decay of the baryon singlet $S^{0} \rightarrow \Sigma \pi$ involves no $\gamma^{5}$ as appropriate in our conventions for a baryon with opposite parity

$$
\begin{aligned}
\mathcal{L}_{S \Sigma \pi} & =-\frac{i}{\sqrt{3}} \operatorname{Tr}\left\{\bar{N}_{8} \tilde{a}_{\mu}\right\} \gamma^{\mu} S^{0}+\text { h.c. } \\
& =-\frac{i}{\sqrt{6} f_{\pi}}\left(\bar{\Sigma}^{+} \partial_{\mu} \pi^{+}+\bar{\Sigma}^{-} \partial_{\mu} \pi^{-}+\bar{\Sigma}^{0} \partial_{\mu} \pi^{0}\right) \gamma^{\mu} S^{0}+\text { h.c. }
\end{aligned}
$$

It involves no additional parameter such that the decay width is computable.

For a discussion of the self-interactions of the pseudoscalar mesons we first expand eq. (91) in powers of $\Pi=\frac{1}{2} \Pi^{z} \lambda_{z}$

$$
\begin{aligned}
\mathcal{L}^{(\pi)} & =\frac{f^{2}}{4} \operatorname{Tr}\left\{\partial^{\mu} \exp \left(-\frac{2 i}{f} \Pi\right) \partial_{\mu} \exp \left(\frac{2 i}{f} \Pi\right)\right\} \\
& =\operatorname{Tr}\left\{\partial^{\mu} \Pi \partial_{\mu} \Pi\right\}+\frac{1}{f^{2}} \operatorname{Tr}\left\{\partial^{\mu} \Pi^{2} \partial_{\mu} \Pi^{2}-\frac{4}{3} \partial^{\mu} \Pi \partial_{\mu} \Pi^{3}\right\}+\ldots
\end{aligned}
$$

Similarly, the current quark mass term (96) contributes

$$
\begin{aligned}
\mathcal{L}_{j} & =\operatorname{Tr}\left\{M_{(p)}^{2} \Pi^{2}\right\}-\operatorname{Tr}\left\{\frac{M_{(p)}^{2}}{3 f^{2}} \Pi^{4}\right\}+\ldots \\
M_{(p)}^{2} & =2 Z_{\phi}^{-1 / 2} a_{q} \sigma_{0} f^{-2} \bar{m} .
\end{aligned}
$$

This yields the low-momentum four-pion interactions. Further effective interactions arise from the exchange of vector mesons according to eq. (93). They are obtained by substituting in $\mathcal{L}$ for the vector meson fields $\tilde{V}_{\mu}$ the solution of the field equation in presence of pseudoscalars

$$
\tilde{V}_{\mu}=-\frac{\bar{M}_{\rho}^{2}}{g} G_{\mu}^{(\rho) \nu} \tilde{v}_{\nu}+\ldots
$$

Here the vector meson propagator $G^{(\rho)}$ obeys

$$
G_{\mu}^{(\rho) \nu}\left[\left(\bar{M}_{\rho}^{2}-\partial^{2}\right) \delta_{\nu}^{\sigma}+\partial_{\nu} \partial^{\sigma}\right]=\delta_{\mu}^{\sigma} .
$$

One obtains

$$
\begin{aligned}
\mathcal{L}_{V}= & \frac{1}{16 g^{2}} \operatorname{Tr}\left\{\partial_{\mu} U^{\dagger} \partial^{\mu} U \partial_{\nu} U^{\dagger} \partial^{\nu} U-\partial_{\mu} U^{\dagger} \partial_{\nu} U \partial^{\mu} U^{\dagger} \partial^{\nu} U\right\} \\
= & \frac{1}{32 g^{2}}\left[6 \operatorname{Tr}\left\{\partial_{\mu} U^{\dagger} \partial^{\mu} U \partial_{\nu} U^{\dagger} \partial^{\nu} U\right\}-\left(\operatorname{Tr}\left\{\partial_{\mu} U^{\dagger} \partial^{\mu} U\right\}\right)^{2}\right. \\
& \left.-2 \operatorname{Tr}\left\{\partial_{\mu} U^{\dagger} \partial_{\nu} U\right\} \operatorname{Tr}\left\{\partial^{\mu} U^{\dagger} \partial^{\nu} U\right\}\right] .
\end{aligned}
$$


From eq. (138) one can infer the contribution of $\mathcal{L}_{V}$ to the parameters $L_{i}$ appearing in next to leading order in chiral perturbation theory [21]

$$
L_{1}^{(V)}=\frac{1}{32 g^{2}} \quad, \quad L_{2}^{(V)}=\frac{1}{16 g^{2}} \quad, \quad L_{3}^{(V)}=-\frac{3}{16 g^{2}} .
$$

We observe that the constants depend only on the effective gauge coupling $g$. For $g=6$ the values $L_{1}^{(V)}=0.87 \cdot 10^{-3}, L_{2}^{(V)}=1.74 \cdot 10^{-3}, L_{3}^{(V)}=-5.2 \cdot 10^{-3}$ compare well with the values [22] extracted from observation $L_{1}=(0.7 \pm$ $0.3) \cdot 10^{-3}, L_{2}=(1.7 \pm 0.7) \cdot 10^{-3}, L_{3}=-(4.4 \pm 2.5) \cdot 10^{-3}$. This is consistent with the hypothesis that these constants are dominated ${ }^{15}$ by vector-meson exchange [24. The momentum dependence of the effective four-pion vertex extracted from inserting eq. (136) describes the fact that the $\pi-\pi$ scattering at intermediate energies is dominated by the $\rho$-resonance. We conclude that the simple effective action (42) gives a very satisfactory picture of the pion interactions even beyond lowest order in chiral perturbation theory!

\section{Conclusions and Discussion}

The "spontaneous breaking" of color seems to be compatible with observation. The simple effective action (42) gives a realistic approximate description of the masses of all low-lying mesons and baryons and of their interactions. Gluon-meson duality is associated to the well-known Higgs phenomenon with colored composite scalar fields, corresponding to quark-antiquark pairs.

The most important characteristics of our scenario can be summarized in the following points.

(i) The Higgs mechanism generates a mass for the gluons. In the limit of equal (current) masses for the three light quarks the masses of all gluons are equal. The massive gluons transform as an octet with respect to the physical SU(3)-symmetry. They carry integer electric charge, isospin and strangeness and can be identified with the vector meson octet $\rho, K^{*}, \omega$.

(ii) The physical fermion fields are massive baryons with integer electric charge. The low mass baryons form an octet with respect to the $S U(3)$ symmetry group of the "eightfold way". In a gauge-invariant language the baryons are quarks with a dressing of nonlinear fields. In a gauge-fixed version quarks and baryons can be described by the same field. This is quark-baryon duality. The main contribution to the mass of these baryons arises from chiral symmetry breaking through quark-antiquark condensates in the color

\footnotetext{
${ }^{15}$ Further contributions arise from the exchange of scalars and have been estimated [23] as $L_{2}^{(S)}=0, L_{3}^{(S)}=1.3 \cdot 10^{-3}$.
} 
singlet and octet channels. These considerations extend [1] to the heavy quarks $c, b, t$, except that the mass is now dominated by the current quark mass. The lightest charmed baryons (and t-baryons) belong to a $S U(3)$ antitriplet with electric charge 0,1,1. Correspondingly, the $S U(3)$-antitriplet of the lightest $b$-baryons carries electric charge $-1,0,0$.

(iii) Our scenario shares the properties of chiral symmetry breaking with many other approaches to long-distance strong interactions. In particular, chiral perturbation theory is recovered in the low energy limit. This guarantees the observed mass pattern for pions, kaons and the $\eta$-meson and the structure of their (low momentum) interactions.

(iv) Spontaneous color symmetry breaking generates a nonlinear local $S U(3)_{P}$-reparameterization symmetry. The gauge bosons of this symmetry originate from the gluons. They form the octet of low masses physical vector mesons. The symmetry relations following from $S U(3)_{P}$ symmetry appear in the electromagnetic and strong interactions of the vector mesons and lead to vector dominance. They are compatible with observation and provide for a successful test of our scenario.

(v) Weak interactions can be incorporated naturally in our framework [1]. The $\Delta I=1 / 2$ rule for the weak hadronic kaon decays turns out to be a consequence of the properties of the lowest dimension operators which are consistent with the symmetries.

Beyond the important general symmetry relations arising from chiral symmetry and the nonlinear local reparameterization symmetry the simple effective action (42) leads to particular predictions. They are related to the assumption that the effective action can be described in leading order by effective couplings with positive or zero mass dimension. (This counting holds if composite scalar fields are counted according to the canonical dimension for scalar fields.) For this purpose we fix the parameters $\chi_{0}, \sigma_{0}$ and $g$ by $M_{\rho}, f_{\pi}$ and $\Gamma\left(\rho \rightarrow e^{+} e^{-}\right)$. More precisely, we use here eqs. (5.16), (5.17) (7.14) with $\bar{M}_{\rho}=850 \mathrm{MeV}, f_{0}=128 \mathrm{MeV}, g_{\rho \gamma}=0.12 \mathrm{GeV}^{2}$. In addition, the Yukawa couplings $h, \tilde{h}$ are fixed by the baryon masses $M_{8}, M_{1}$ (cf. eq. (4.10)) whereas the strength of the chiral anomaly $\nu$ and $\nu^{\prime}$ determines $M_{\eta^{\prime}}^{2}$ by eq. (5.19). (The precise ratio $\nu^{\prime} / \nu$ is not relevant here.) For equal quark masses we concentrate on the following predictions of eq. (42) which are not dictated by symmetry considerations:

(1) The pion nucleon couplings are found as $F=D=0.5$ to be compared with the observed values $F=0.459 \pm 0.008, D=0.798 \pm 0.008$.

(2) The decay width $\Gamma(\rho \rightarrow 2 \pi)=115 \mathrm{MeV}$ turns out to be somewhat lower than the observed value of $150 \mathrm{MeV}$. (Note that we use here directly eq. (118) and $\kappa_{f}$ equals one in leading order, such that $g_{\rho \pi \pi}=4.6$.)

(3) The direct coupling of the photon to pions is suppressed $g_{\gamma \pi \pi} / e=0.04$ 
(by virtue of eq. (110) with $M_{\rho} \rightarrow \bar{M}_{\rho}, f_{\pi} \rightarrow f_{0}$ ). This phenomenon of vector dominance describes well the observations.

(4) The effective next-to leading order couplings $L_{1}, L_{2}, L_{3}$ of chiral perturbation theory come out compatible with observation $L_{2}=1.7 \cdot 10^{-3}, L_{3}=$ $-(3.9-5.2) \cdot 10^{-3}$.

For a first approximation we consider the effective action (42) as very satisfactory. Corrections arise from two sources: (a) The nonequal quark masses break the physical global $S U(3)$-symmetry. (b) Higher order invariants are certainly present in the full effective action. Some of the corrections are known phenomenologically as, for example, the partial Higgs effect which reduces the average meson decay constant from $f_{0}$ to the mean value $f=\left(f_{\pi}+2 f_{K}\right) / 3=106 \mathrm{MeV}$. Together with the further lowering of $f_{\pi}$ as compared to $f$ by $S U(3)_{V}$ violation this leads to a realistic value of $\Gamma(\rho \rightarrow 2 \pi)$. The neglected higher-order invariants can be used to improve the agreement with observation. Typically, these corrections are below $30 \%$.

Many more observable quantities could be computed from the effective action (42), especially if we take into account the effects of $S U(3)$-violation due to the strange quark mass [25]. (This will involve additional parameters in the effective potential beyond $\sigma_{0}$ and $\chi_{0}$.) We will rather give here an outlook on some key issues for future theoretical developments. At the end, the parameters of the effective action (42) should be computed from the microscopic action of QCD. Their values can only depend on the strong gauge coupling (or $\Lambda_{Q C D}$ ) and the quark masses. A first attempt based on an instanton computation [26] gives a rather satisfactory result for the value of the gluon/vector meson mass $M_{\rho}$ and the associated value of the octet condensate $\chi_{0}$. It also yields reasonable values for the $<\bar{q} q>$-condensate in the singlet channel and for the mass and two-photon-decay width of the $\eta^{\prime}$-meson.

An interesting question concerns a variation of the quark masses. For a large value of the strange quark mass $m_{s}$ (and small up and down quark mass) an interesting Higgs picture of QCD for $N_{f}=2$ has been proposed in 27. If all quark masses get heavy there should be a transition to gluodynamics $\left(N_{f}=0\right)$ which presumably is characterized by an effective infrared cutoff different from the octet condensate relevant for $N_{f}=3$. This raises interesting issues for the behavior of the heavy quark potential as the quark mass is varied [25] which can perhaps be tested by lattice simulations. Other possible lattice tests concern the equivalence of the Higgs and confinement pictures. One may introduce "fundamental" scalar octets with the transformation of $\chi$ together with a classical potential. This would allow one to move continuously from the Higgs phase with a value of $\chi_{0}$ independent of QCD to the QCD universality class which can be realized in this setting by 
a large positive mass term in the classical potential for $\chi$. For this purpose one could vary the octet mass term from large negative to large positive values. A smooth transition form the Higgs phase to the QCD-universality class would be a strong argument in favor of the possibility of a Higgs description for the vacuum of real QCD.

Finally, our approach has all ingredients for an analytical investigation of the QCD-phase diagram. One and the same model can describe both quarks and gluons at high temperature $T$ and hadrons for low $T$. The crucial qualitative change at the high temperature phase transition (for small baryon density) is the formation of the octet condensate as the temperature is lowered below the critical temperature $T_{c}$. For $T>T_{c}$ one has $\chi_{0}=0$ and $\sigma_{0}$ takes a small value which vanishes in the chiral limit of vanishing quark masses. Both gluons and quarks are massless. In contrast, the hadronic phase at $T<T_{c}$ is characterized by nonvanishing $\chi_{0}, \sigma_{0}$ and resembles the vacuum described in this work. Since the "octet melting" for $T>T_{c}$ accounts simultaneously for both the quarks and the gluons becoming massless ${ }^{16}$ one finds a convincing explanation for the equality of the "chiral restoration temperature" and the "deconfinement temperature" observed in lattice simulations. A first quantitative computation of $T_{c}$ in our model (mean field calculation) yields $T_{c} \approx 170 \mathrm{MeV}[29$.

A similar computation for a nonvanishing baryon chemical potential still needs to be done. Recently a strong argument has advocated that the critical temperature equals the chemical freezeout temperature and therefore has been measured experimentally [30]. An extrapolation of this argument to the experiments at large baryon density (AGS) suggests a very simple phase diagram for QCD: A first order line separates the high-temperature quark gluon plasma (in our picture $\chi_{0}=0, \sigma_{0}$ small) form the hadron gas $\left(\chi_{0}, \sigma_{0}\right.$ close to vacuum values). This line coincides for small $T$ and large baryon density $\left(\mu_{B} \approx m_{n}\right)$ with the first order line between the hadron gas and nuclear matter (nuclear gas and nuclear liquid). Nuclear matter for $T=0$ is characterized by an additional diquark condensate $\left\langle d_{3} d_{3}>[25\right.$ ] which spontaneously breaks the global symmetries of baryon number and isospin while conserving strangeness. In the dual hadronic description of our model this diquark condensate corresponds to a neutron-neutron condensate. $\mathrm{Nu}-$ clear matter (or the nuclear liquid) is superfluid due to the Goldstone boson associated with the spontaneous breaking of the third component of isospin $I_{3}$. (Neglecting the up and down quark masses and electroweak interactions one would have three Goldstone bosons [25] associated with the spontaneous breaking of global $S U(2)$-isospin symmetry.) The existence of a symmetry

\footnotetext{
${ }^{16}$ This issue has been related to the idea of "vector manifestation" [28].
} 
breaking order parameter implies that the nuclear matter phase is separated by a true phase transition line from the quark gluon plasma at high temperature and high baryon density. This phase transition may be second order in the $O(2)$-universality class. Critical fluctuations could characterize the point where this line meets the first order transition between the quark gluon plasma and the hadron gas. (Often critical fluctuations are associated to an endpoint of the transition between a nuclear liquid and a nuclear gas.) Finally, for low temperature and baryon density substantially larger than nuclear density one expects a further transition to a "color-flavor-locked" color superconductor [7]. This is distinguished from nuclear matter by (approximate) $S U(3)$ symmetry. The superfluidity in this phase originates form the spontaneous breaking of baryon number while $I_{3}$ remains unbroken. In the limit of equal quark masses $m_{s}=m_{d}=m_{u}$ the separate phase of nuclear matter with broken $I_{3}$ disappears and the color superconducting phase connects directly to the hadron gas, with an octet condensate present in both phases [1].

Substantial work remains to be done before the Higgs picture of the QCD vacuum can finally be accepted. Our hypothesis could be falsified if it leads to a direct conflict with the confinement picture, lattice simulations or observation. A better understanding of the nonlinear fields $v, W_{L}, W_{R}$ relating quarks to baryons is needed in order to establish the connection to the successful picture of the nonrelativistic quark model and to address basic issues like the structure functions in high energy hadron scattering. 


\section{References}

[1] C. Wetterich, Phys. Rev. D64 (2001) 036003

[2] G. 't Hooft, in: Recent Developments in Gauge Theories (Plenum, New York, 1980), p. 135;

S. Dimopoulos, S. Raby, L. Susskind, Nucl. Phys. B173 (1980) 208;

T. Matsumoto, Phys. Lett. 97B (1980) 131;

M. Yasuè, Phys. Rev. D42 (1990) 3169

[3] T. Banks, E. Rabinovici, Nucl. Phys. B160 (1979) 349;

E. Fradkin, S. Shenker, Phys. Rev. D19 (1979) 3682

[4] M. Reuter, C. Wetterich, Nucl. Phys. B408 (1993) 91;

C. Wetterich, "Electroweak Physics and the Early Universe", eds. J. Romao and F. Freire, Plenum Press (1994) 229;

W. Buchmüller, O. Philipsen, Nucl. Phys. B443 (1995) 47;

K. Kajantie, M. Laine, R. Rummukainen, M. Shaposhnikov, Phys. Rev. Lett. 77 (1996) 2887;

for a review see B. Bergerhoff, C. Wetterich, hep-ph/9611462, in "Current topics in astrofundamental physics", eds. N. Sanchez and A. Zichichi, p. 132, World Scientific 1997

[5] P. Damgaard, U. Heller, Phys. Lett. 171 B (1986), 442; Nucl. Phys. B294 (1987) 253; B304 (1988) 63;

H. Evertz, J. Jersak, K. Kanaya, Nucl. Phys. B285 (1987) 229

[6] C. Wetterich, Z. Phys. C57 (1993) 451

[7] D. Bailin, A. Love, Phys. Rep. 107 (1984) 325;

M. Alford, K. Rajagopal, F. Wilczek, Phys. Lett. 422B (1998) 247;

Nucl. Phys. B537 (1999) 443;

R. Rapp, T. Schäfer, E. Shuryak, M. Velkovsky, Phys. Rev. Lett. 81 (1998) 53;

J. Berges, K. Rajagopal, Nucl. Phys. B538 (1999) 214

[8] T. Schäfer, F. Wilczek, Phys. Rev. Lett. 82 (1999) 3956;

M. Alford, J. Berges, K. Rajagopal, Nucl. Phys. B558 (1999) 219

[9] J. Hubbard, Phys. Rev. Lett. 3 (1959) 77;

R. Stratonovich, Dokl. Akad. Nauk. SSR 115 (1957) 1097

[10] E. Meggiolaro, C. Wetterich, Nucl. Phys. B606 (2001) 337 
[11] H. Gies, C. Wetterich, Phys. Rev. D69 (2004) 025001

[12] C. Wetterich, Z. Phys. C48 (1990) 693

[13] G. 't Hooft, Phys. Rev. D14 (1976) 3432;

M. Shifman, A. Vainshtein, V. Zakharov, Nucl. Phys. B163 (1980) 46;

M. Nowak, J. Verbaarschot, I. Zahed, Nucl. Phys. B324 (1989) 1;

T. Schäfer, E. Shuryak, Rev. Mod. Phys. 70 (1998) 323

[14] D. Jungnickel, C. Wetterich, Phys. Rev. D53 (1996) 5142;

J. Berges, D. Jungnickel, C. Wetterich, Phys. Rev. D59 (1999) 034010

[15] J. Jaeckel, C. Wetterich, Nucl. Phys. A733 (2004) 113

[16] D. Jungnickel, C. Wetterich, Eur. Phys. J. C1 (1998) 669

[17] D. Jungnickel, C. Wetterich, Phys. Lett. B389 (1996) 600

[18] M. Bando, T. Kugo, K. Yamawaki, Phys. Rep. 164 (1988) 217

[19] K. Kawarabayahi, M. Suzuki, Phys. Rev. Lett 16 (1966) 255;

Riazuddin and Fayyazuddin, Phys. Rev. 147 (1961) 1071

[20] B. Serot, D. Walecka, Int. J. Mod. Phys. E6 (1997) 515

[21] J. Gasser, H. Leutwyler, Phys. Rep. C87 (1982) 77; Nucl. Phys. B250 (1985) 465

[22] J. Bijnens, J. G. Ecker, J. Gasser, DAPHNE physics handbook, hep-ph/9411232

[23] D. Jungnickel, C. Wetterich, Eur. Phys. J. C2 (1998) 557

[24] J. G. Ecker, J. Gasser, A. Pich, E. de Rafael, Nucl. Phys. B321 (1989) 311

[25] C. Wetterich, Eur. Phys. J. C29 (2003) 251

[26] C. Wetterich, Phys. Lett. B525 (2002) 277

[27] J. Berges, C. Wetterich, Phys. Lett. B512 (2001) 85

[28] M. Rho, hep-ph/0303136

[29] C. Wetterich, Phys. Rev. D66 (2002) 056003

[30] P. Braun-Munzinger, J. Stachel, C. Wetterich, Phys. Lett. B596 (2004) 105008 